ARTICLE

\title{
Positive feedback regulation of p53 transactivity by DNA damage-induced ISG15 modification
}

Jong Ho Park ${ }^{1, \star}$, Seung Wook Yang ${ }^{1, \star}$, Jung Mi Park ${ }^{1}$, Seung Hyeun Ka ${ }^{1}$ Ji-Hoon Kim ${ }^{1}$, Young-Yun Kong ${ }^{1}$, Young Joo Jeon ${ }^{2}$, Jae Hong Seol ${ }^{1} \&$ Chin Ha Chung ${ }^{1,3}$

p53 plays a pivotal role in tumour suppression under stresses, such as DNA damage. ISG15 has been implicated in the control of tumorigenesis. Intriguingly, the expression of ISG15, UBE1L and UBCH8 is induced by DNA-damaging agents, such as ultraviolet and doxorubicin, which are known to induce p53. Here, we show that the genes encoding ISG15, UBE1L, $\mathrm{UBCH} 8$ and EFP, have the p53-responsive elements and their expression is induced in a p53dependent fashion under DNA damage conditions. Furthermore, DNA damage induces ISG15 conjugation to $\mathrm{p} 53$ and this modification markedly enhances the binding of $\mathrm{p} 53$ to the promoters of its target genes (for example, CDKN1 and BAX) as well as of its own gene by promoting phosphorylation and acetylation, leading to suppression of cell growth and tumorigenesis. These findings establish a novel feedback circuit between p53 and ISG15-conjugating system for positive regulation of the tumour suppressive function of p53 under DNA damage conditions.

\footnotetext{
${ }^{1}$ School of Biological Sciences, College of Natural Sciences, Seoul National University, Seoul 08826, Korea. ${ }^{2}$ Department of Biochemistry, Institute of Medical Science, Chungnam National University School of Medicine, Daejon 34134, Korea. ${ }^{3}$ The 2nd Division of Natural Sciences, National Academy of Science, Seoul 06579, Korea. ${ }^{*}$ These authors contributed equally to this work. Correspondence and requests for materials should be addressed to J.H.S. (email: jhseol@snu.ac.kr) or to C.H.C. (email: chchung@snu.ac.kr).
} 
T he p53 tumour suppressor has been regarded as 'guardian

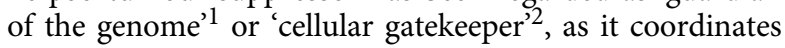
cellular responses to various stress signals, such as DNA damage, abnormal oncogene activation, telomere erosion and hypoxia ${ }^{3,4}$. Under normal conditions, p53 is downregulated by several ubiquitin E3 ligases, including the major MDM2 ligase, and subsequent degradation by proteasome. Notably, the expression of MDM2 is induced by p53, thus forming a negative feedback loop for keeping p53 at a low level ${ }^{5-7}$. Under stressed conditions, however, p53 is stabilized and activated by disruption of its interaction with MDM2 and the other negative regulators through phosphorylation and acetylation. The activated p53 then binds to a specific DNA sequence, called the p53-responsive element ( $p 53 R E)$, for transcriptional activation of its target genes (for example, CDKN1, BAX and PUMA) that mediate cell cycle arrest and apoptosis ${ }^{8-10}$.

Since p53 is involved in the control of numerous critical cellular processes, its transactivity needs to be tightly regulated ${ }^{11}$. The p53 activity is regulated by a wide variety of post-translational modifications, including the modification by ubiquitinlike proteins, in addition to phosphorylation, methylation, acetylation and ubiquitination. MDM2- and FBXO11-mediated neddylation inhibits p53 transcriptional activity ${ }^{12,13}$, whereas sumoylation promotes it $^{14,15}$. Recently, it has been reported that ISG15, the product of the interferon (IFN)-stimulated gene 15 , can be conjugated primarily to misfolded p 53 and this modification promotes the degradation of p53 by proteasome $\mathrm{e}^{16}$. However, it remains unknown when and how the modification of p53 by ubiquitin-like proteins occurs for the control of the p53 activity.

ISG15 is the first reported ubiquitin-like protein ${ }^{17}$. ISG15 expression is robustly induced by type-I IFNs, lipopolysaccharides and viral infection ${ }^{18,19}$. Like ubiquitination, protein ISGylation is catalysed by a three-step enzyme system: UBE1L as an ISG15-activating E1 enzyme, UBCH8 as an ISG15conjugating E2 enzyme and EFP and HERC5 as ISG15 E3 ligases ${ }^{19-22}$. This protein ISGylation can be reversed by an ISG15deconjugating enzyme, UBP43 also called USP18 (ref. 23). In addition to conjugation to target proteins, type-I IFN-induced ISG15 is secreted from leukocytes, such as monocytes and lymphocytes, and serves as a cytokine that stimulates synthesis and secretion of IFN- $\gamma^{24,25}$.

Numerous studies using murine system have demonstrated that protein modification by ISG15 mediates anti-viral responses. Mice lacking Ube1L exhibit increased susceptibility to influenza B virus infection ${ }^{26}$ and ISG15-deficient mice are more susceptible to influenza $A$ and $B$, Sindbis and herpes virus infections ${ }^{27}$. In addition, a loss-of-function mutation within the Usp18 gene $\left(U_{\left.s p 18^{l t y 9}\right)}\right.$ in mice confers increased susceptibility to Salmonella Typhimurium $^{28}$. On the other hand, in human, free ISG15 secreted from granulocytes plays a crucial role as an IFN- $\gamma$ inducing cytokine for optimal antimycobacterial immunity ${ }^{29,30}$, while intracellular ISG15 functions in UBP43-mediated downregulation of type-I IFN signalling and prevention of typeI IFN-dependent auto-inflammation ${ }^{31}$. Remarkably, ISG15 deficiency in human, unlike in mice, causes little or no change in susceptibility to viral infection ${ }^{29,30}$, indicating distinctive as well as diverse roles of ISG15 in mammals.

Protein ISGylation and de-ISGylation appear to also function in the control of DNA damage responses. We have recently shown that DNA-damaging agents, such as doxorubicin, induce ISGylation of the oncogenic $\Delta \mathrm{Np} 63 \alpha$ protein for suppression of epithelial tumours ${ }^{32}$. We also have shown that ultraviolet induces ISGylation of PCNA for termination of DNA damage-induced error-prone translesion synthesis for maintaining genome stability $^{33}$. Notably, during these studies, we found that the expression of ISG15, UBE1L and UBCH8 can be induced by DNA-damaging agents, such as ultraviolet, doxorubicin and camptothecin, all of which are known to induce p53. These findings raised a possibility that DNA damage-induced expression of the ISG15-conjugating machinery is under the control of p53.

In the present study, we show that all of the genes encoding ISG15, UBE1L, UBCH8 and EFP have p53REs in the promoter regions for their p53-mediated expression under DNA damage conditions. Remarkably, p53 serves as a target for ISGylation and this modification dramatically increased the binding of $\mathrm{p} 53$ to the promoter regions of its target genes as well as of its own gene, forming a positive feedback loop, which would amplify the expression of the genes for cell cycle arrest and apoptosis, such as CDKN1 and BAX. Collectively, these results indicate that ISGylation of p53 plays a critical role in cell growth inhibition and thereby in suppression of tumour development under DNA damage conditions.

\section{Results}

p53 induces the expression of ISG15-conjugating system. We have recently shown that DNA-damaging agents, such as doxorubicin, camptothecin or ultraviolet, induce the expression of both messenger RNA (mRNA) and protein levels of ISG15, UBE1L and UBCH8 (refs 32,33). These findings suggest that the expression of ISG15, UBE1L and UBCH8 may be regulated by p53 via DNA damage-induced activation of ATM and ATR kinases. To test this possibility, $p 53^{+/+}$HCT116 cells (human colon carcinoma) were incubated with caffeine, an ATR and ATM inhibitor ${ }^{34}$, immediately after treatment with the DNA-damaging agents (Fig. 1). As expected, caffeine abrogated phosphorylation of Chk1 and p53 and thereby the expression of p53. Remarkably, the drug also strongly inhibited the expression of EFP as well as of ISG15, UBE1L and UBCH8 (all together henceforth referred to as ISG15-conjugating system). Supplementary Figure 1 shows the quantitative data for the changes in the levels of ISG15-conjugating system in the presence and absence of caffeine under DNA damage conditions. Note that we examined the effect of caffeine on EFP expression, since of two known ISG15 E3 ligases, EFP, but not HERC5, interacts with p53 for ISGylation (see below). Similar results were obtained when caffeine was treated to other cancer cells, including U2OS (human osteosarcoma), MCF7 (human breast carcinoma) and A549 (human lung carcinoma), all of which are known to express normal p53 (Supplementary Fig. 2). One exception, however, was HeLa cells (human cervical carcinoma): caffeine showed relatively little effect on the expression of ISG15, although it inhibited that of UBE1L, UBCH8 and EFP (Supplementary Fig. 3). These results suggest that the expression of ISG15-conjugating system is regulated by $\mathrm{p} 53$, although it remains unclear how DNA damageinduced expression of ISG15 in HeLa cells could occur in the presence of caffeine.

To verify further p53-mediated induction of ISG15-conjugating system, we compared their expression in $p 53^{+1+}$ and $p 53^{-/}$- cells. Ultraviolet treatment to $p 53^{-/-}$cells showed little or no effect on the expression of ISG15-conjugating system, unlike that to $p 53^{+/+}$cells (Supplementary Fig. 4a,b). Furthermore, the expression of a p53-specific short hairpin RNA (shRNA; shp53), but not a nonspecific shRNA (shNS), in $p 53^{+1+}$ cells abrogated ultraviolet-induced expression of ISG15-conjugating system (Supplementary Fig. 4c,d). Similar results were obtained when doxorubicin or camptothecin was treated in place of ultraviolet. Taken together, these results indicate that the expression of ISG15-conjugating system is upregulated by $\mathrm{p} 53$ under DNA damage conditions. 
a

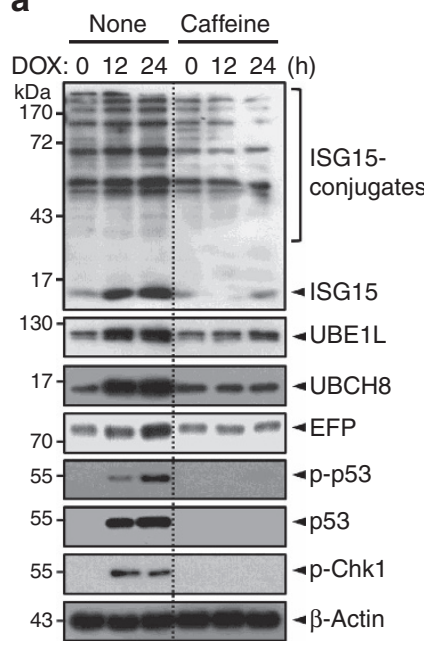

b

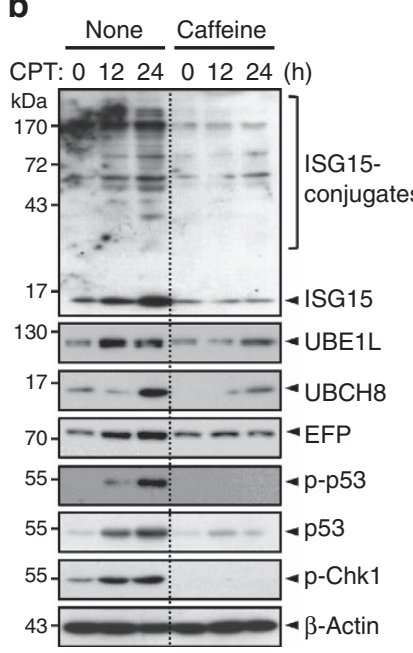

C

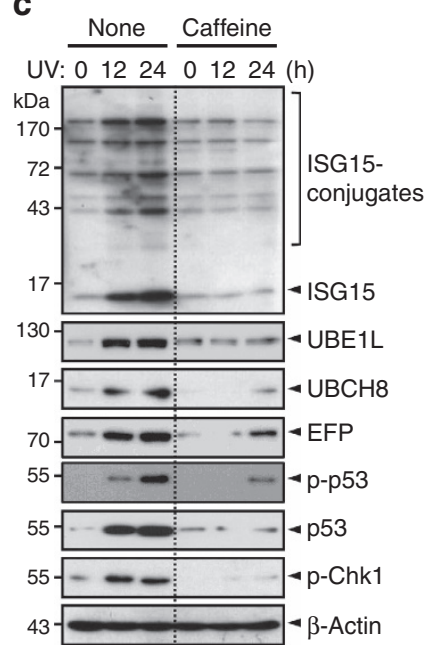

Figure 1 | ATM/ATR kinases induce the expression of ISG15-conjugating system. $p 53^{+/+}$HCT116 cells were treated with $1 \mu M$ doxorubicin (DOX) (a), $0.25 \mu \mathrm{M}$ camptothecin (CPT) (b) and $30 \mathrm{~J} \mathrm{~m}^{-2}$ ultraviolet (UV) (c). Immediately after the treatment, the cells were incubated with $5 \mathrm{mM}$ caffeine for increasing periods. The cells were washed with iced PBS, and lysed in $50 \mathrm{mM}$ Tris- $\mathrm{HCl}(\mathrm{pH} 7.4), 150 \mathrm{mM} \mathrm{NaCl}, 1 \mathrm{mM}$ EDTA, $1 \mathrm{mM}$ NEM, $1 \mathrm{mM}$ sodium orthovanadate, $1 \mathrm{mM} \mathrm{NaF}, 1 \mathrm{mM}$ PMSF, $0.2 \%(\mathrm{v} / \mathrm{v})$ Triton $\mathrm{X}-100$ and $1 \mathrm{X}$ protease inhibitor cocktail. The samples were centrifuged for 30 min at $16,000 \mathrm{~g}$ and the supernatant fractions (cell lysates) were subjected to immunoblot with anti-ISG15, anti-UBE1L anti-UBCH8, anti-EFP, anti-phospho-p53 (p-p53), anti-p53, anti-phospho-Chk1 ( $p$-Chk1) and anti- $\beta$-actin antibodies (from the top to the bottom, respectively).

The genes encoding ISG15-conjugating system has p53REs. We next examined whether the genes encoding ISG15-conjugating system have the p53-response elements ( $p 53 R E s)$ for their p53-mediated expression. Sequence analysis showed the presence of several candidate sequences (termed $R E 1$ to $R E 4$ ) in the promoter regions of the ISG15, UBE1L, UBCH8 and EFP genes (Supplementary Fig. 5), which are similar to the known sequence

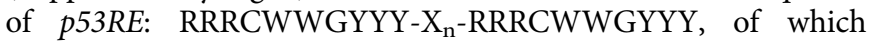
$\mathrm{R}$ stands for purine, $\mathrm{W}$ for adenine or thymine, $\mathrm{Y}$ for pyrimidine and $\mathrm{X}$ for spacer with $n \sim 21$ (ref. 35).

To identify true $p 53 R E(\mathrm{~s})$ among the $R E$ s, serial deletions were generated and fused to a luciferase $(L u c)$ reporter vector (Fig. 2, left panels). The resulting vectors (termed P1 to P4) were expressed in $p 53^{-/-}$HCT116 cells with and without $\mathrm{p} 53$. The p53 ${ }^{+/+}$HCT116 cells were also transfected with P1-P4, followed by treatment with and without ultraviolet. These cells were then subjected to assay for the luciferase activity. The cells transfected with P1-P3 having RE3 of the ISG15 gene, P1 having $R E 1 / R E 2$ of $U B E 1 L, \mathrm{P} 1$ having RE1 of $U B C H 8$, and $\mathrm{P} 1$ having $R E 1 / R E 2$ of $E F P$, but not with the other vectors, showed a significant increase in the luciferase activity upon p53 expression or ultraviolet exposure (Fig. 2, right panels). Furthermore, ChIP analysis revealed that overexpressed p53 in $p 53^{-/-}$HCT116 cells effectively binds to each of the identified $p 53 R E$ s of the genes, as it does to $p 53 R E$ of the $p 21$ gene (Supplementary Fig. 6). These results indicate that the promotor regions of the ISG15, $U B E 1 L, U B C H 8$ and EFP genes have p53REs and their expressions are positively regulated by $\mathrm{p} 53$.

IFN-independent expression of ISG15-conjugating system. Type-I IFNs are known to induce the expression of ISG15conjugating system by activating IFN-stimulated gene factor 3 (ISGF3), which binds to the highly conserved 13-bp sequence of ISRE in their promoters (Supplementary Fig. 5) 21,22,36,37. To determine whether p53 could induce the expression of ISG15conjugating system independently of type-I IFNs, we generated mutations (marked with red in Supplementary Fig. 5) in p53REs and ISREs of $L u c$ reporter vectors for preventing their interaction with p53 and ISGF3, respectively. The mutated vectors (marked by the asterisks in Supplementary Fig. 5) were expressed in p53 $3^{-/-}$HCT116 cells with and without p53 (Fig. 3, left panels). The $p 53^{+/+}$HCT116 cells were also transfected with the same reporter vectors, followed by treatment with and without ultraviolet (Fig. 3, right panels). The mutation of p53REs abrogated p53- and ultraviolet-mediated expression of ISG15conjugating system, but not IFN $\alpha$-mediated expression. On the other hand, the mutation of ISREs prevented IFN $\alpha$-mediated expression of ISG15-conjugating system, but not p53- and ultraviolet-mediated expression. In addition, ultraviolet markedly increased the level of ISGylated cellular proteins only when $p 53^{-/-}$HCT116 cells were supplemented with p53, while IFN $\alpha$ increased it regardless of the presence of p53 (Supplementary Fig. 7). These results indicate that p53 and type-I IFNs can independently induce the expression of ISG15-conjugating system.

To confirm further whether p53 induces the expression of ISG15-conjugating system, p53-null H1299 cells were transfected with an empty plasmid or a vector expressing HisMax-p53. After exposure to ultraviolet, the cells were incubated for increasing periods. The levels of ISG15-conjugating system as well as of ISGylated cellular proteins gradually increased after ultraviolet treatment in cells expressing p53, but not in cells lacking p53 regardless of ultraviolet treatment (Supplementary Fig. 8a). Moreover, the mRNA levels of ISG15-conjugating system increased in a p53-dependent manner under the DNA damage condition (Supplementary Fig. 8b), further demonstrating that p53 positively regulates the expression of ISG15-conjugating system.

DNA damage induces p53 ISGylation. p53 induces MDM2 expression, and MDM2 ubiquitinates p53. Since p53 induces the expression of ISG15-conjugating system, we examined whether p53 could be ISGylated in retrospect. Overexpression of ISG15, UBE1L and UBCH8 in HEK293T cells led to the appearance of a slow-migrating band with the size of $\sim 74 \mathrm{kDa}$ and this band could be disappeared upon co-expression of UBP43, an ISG15deconjugating enzyme (Fig. 4a), indicating that the band represents ISGylated p53. Furthermore, endogenous p53 could be 
a

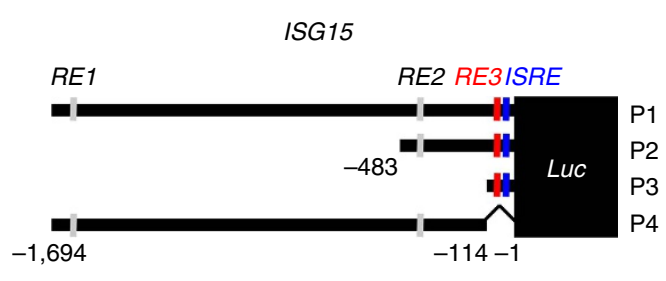

b

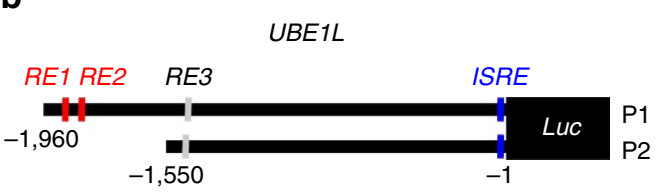

C

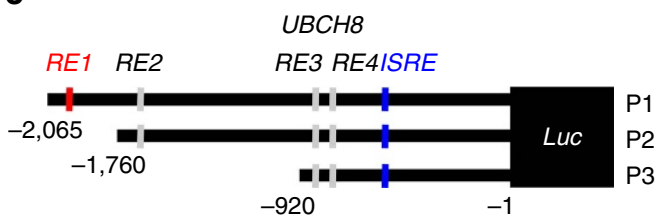

d

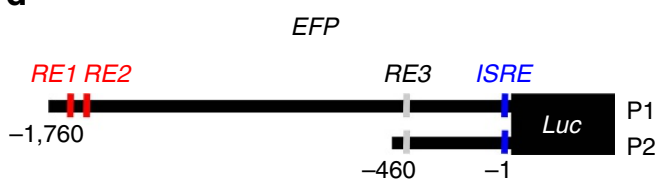

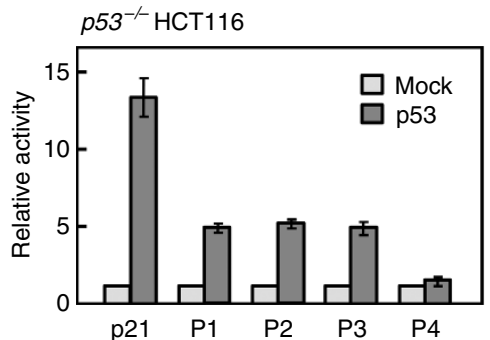

p53 $3^{+/+}$HCT116
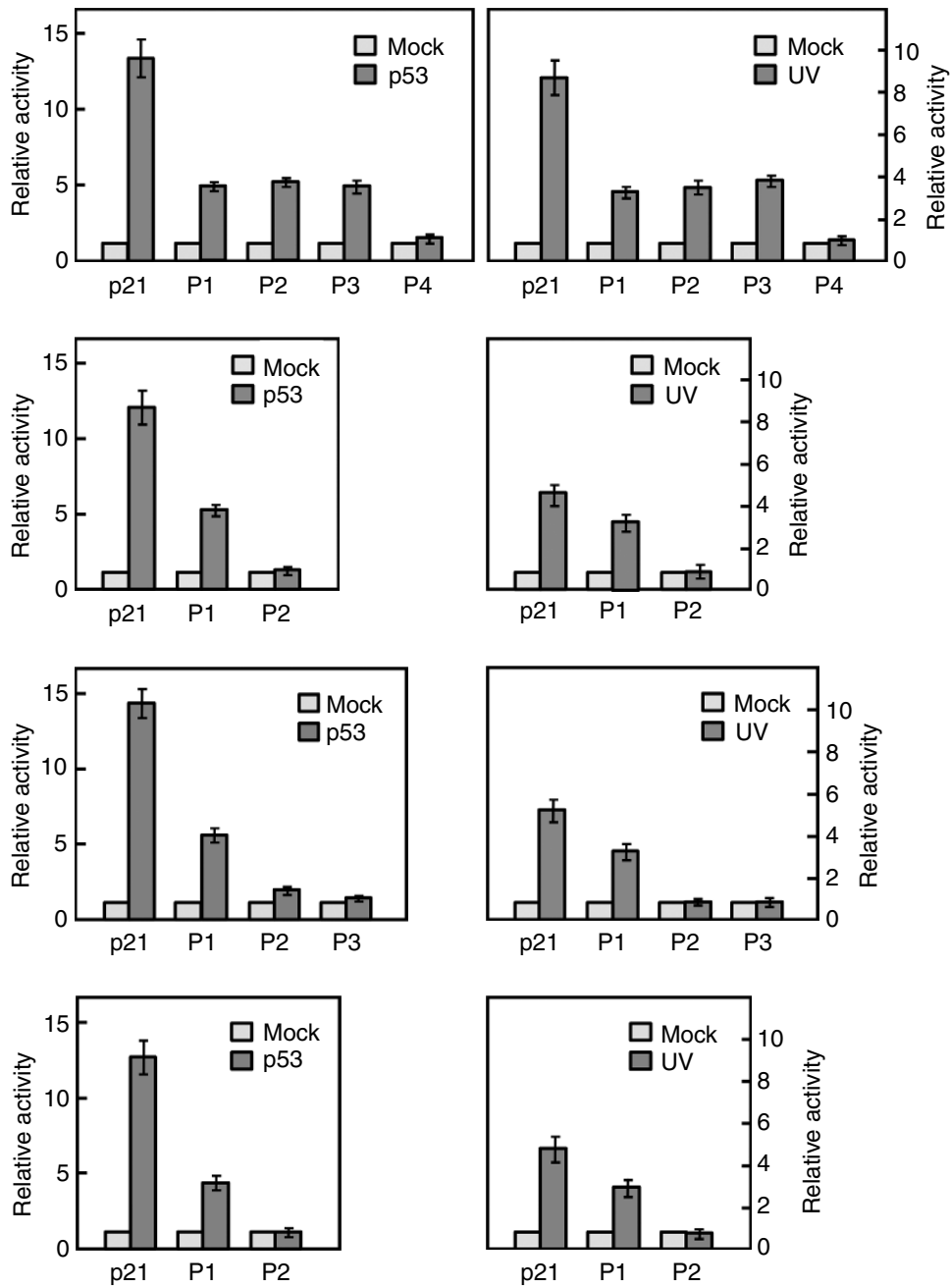

Figure 2 I p53REs in the promoter regions of the genes for ISG15-conjugating system. Deletions of the promoter regions in the ISG15 (a), UBE1L (b), $\mathrm{UBCH} 8$ (c) and EFP genes (d) were fused to a Luc reporter vector ( $\mathrm{dGL3}$ ) (left panels). The resulting vectors (P1-P4) were transfected to p53-/- HCT116 cells with pcDNA-HA-p53. p53 $+/+$ HCT116 cells transfected with the same P1-P4 vectors were treated with and without ultraviolet (UV). p21-Luc was expressed in the cells as a control. These cells were then subjected to assay for the luciferase activity (right panels). The activity seen without any expression or treatment was expressed as 1.0 and the others were expressed as its relative values. Error bar, \pm s.d. $(n=3$ ).

ISGylated upon exposure of $p 53^{+/+}$HCT116 cells to doxorubicin, camptothecin and ultraviolet and this modification could be abolished by the expression of an ISG15-specific shRNA (shISG15), but not by that of shNS (Fig. 4b). These results indicate that p53 serves as an endogenous target for ISGylation under DNA damage conditions.

To identify ISG15 acceptor site(s) in p53, various deletions of p53 (termed P1 to P4) were generated and expressed in HEK293T cells with ISG15, UBE1L and UBCH8. P1 (amino acids 1-300) and P3 (201-393), but not the others, were conjugated by ISG15 (Fig. 4c), indicating that the amino acid sequence 201-300 has ISGylation site(s). Since the 201-300 sequence has two lysine residues (Lys291 and Lys292), each and both of them were replaced by arginine in full-length p 53 by site-directed mutagenesis. The K-to-R mutation of each (K291R or K292R) and both Lys residues (2KR) completely abrogated p53 ISGylation (Fig. 4d), indicating that both Lys291 and Lys292 act as the major ISG15 acceptor sites in p53, although it remains unclear how the mutation of one Lys residue influences ISGylation of the other Lys residue.
To determine whether ISGylation of p53 influences its stability under DNA damage conditions, wild-type p53 and 2KR were expressed in $p 53^{-9}$ HCT116 cells. The cells were treated with and without ultraviolet, and then incubated with cycloheximide for increasing periods. Without ultraviolet treatment, the stability of $2 \mathrm{KR}$ is significantly higher than that of wild-type p53 (Supplementary Fig. 9), consistent with a previous report ${ }^{38}$ showing that both Lys291 and Lys292 serve as the major ubiquitination sites of the MKRN1 ubiquitin E3 ligase, which primarily acts under normal conditions. As expected, ultraviolet treatment led to a dramatic increase in the stability of p53. Notably, however, the $2 \mathrm{KR}$ mutation showed little or no effect on ultraviolet-mediated increase in p53 stability, as if ISGylation of p53 does not influence its stability. Since these experiments were performed under overexpression conditions, we examined whether knockdown of ISG15 influences the stability of endogenous p53 under DNA damage conditions. ISG15 depletion led to a significant, although not dramatic, decrease in p53 stability (Fig. 4e,f). Since ISG15 knockdown markedly reduces the expression of p53 itself and its downstream targets, particularly 
a

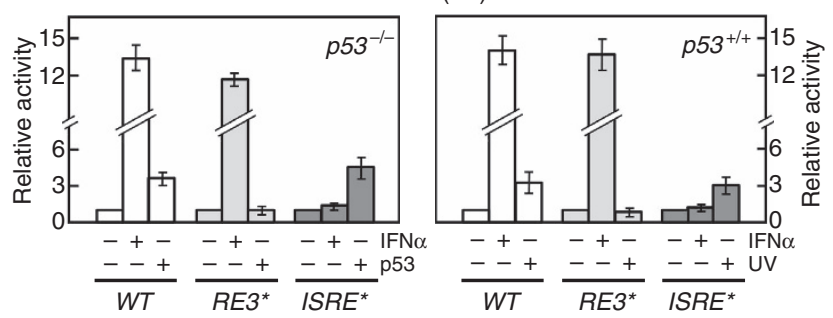

b

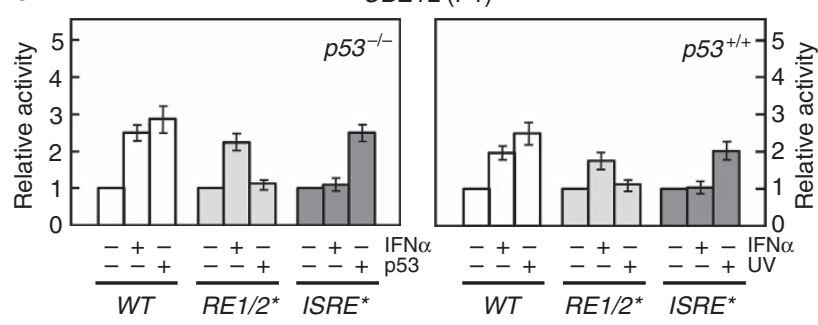

C

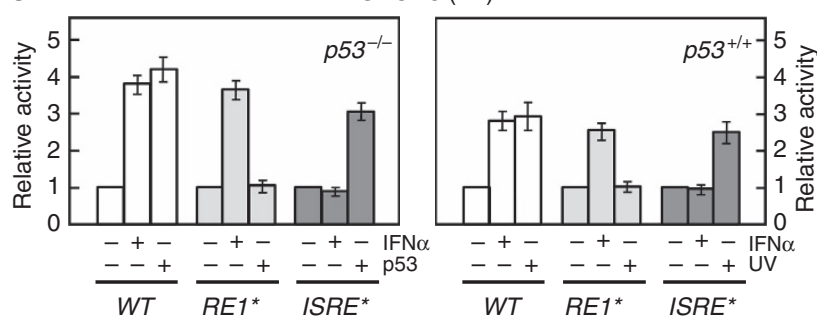

d

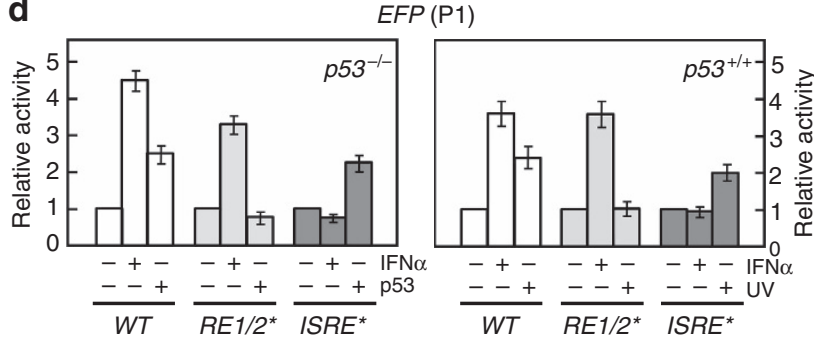

Figure 3 | p53-induced expression of ISG15-conjugating system is independent of type-I IFNs. In P1 reporter vectors (see Fig. 2), RE3 of the ISG15 gene (a), RE1/RE2 of UBE1L (b), RE1 of UBCH8 (c) and RE1/RE2 of EFP (d) were mutated as shown in Supplementary Fig. 5 and the mutated RES were marked by the asterisks. ISREs of the genes were also mutated and marked by the asterisks. After transfection of the vectors, p53 were expressed in $p 53^{-/-}$HCT116 cells or $1,000 \mathrm{U} \mathrm{ml}^{-1}$ of interferon- $\alpha$ (IFN $\left.\alpha\right)$ was treated to the cells (left panels). p53 $1 /+\mathrm{HCT116}$ cells transfected with the same reporter vectors were treated with and without IFN $\alpha$ or ultraviolet (UV; right panels). The cells were then subjected to assay for the luciferase activity. The activity seen without any expression or treatment was expressed as 1.0 and the others were expressed as its relative values. Error bar, \pm s.d. $(n=3)$.

MDM2 (see below), ISG15 depletion-mediated decrease in p53 stability is likely compromised by the reduction in MDM2 expression. Taken together, these results indicate that ISGylation positively regulates the stability of p53 under DNA damage conditions.

EFP serves as a p53-specific ISG15 E3 ligase. EFP and HERC5 have been identified as the major E3 ligases for ISG15 conjugation $^{20,22}$. To identify p53-specific ISG15 E3 ligase among them, we first examined the ability of EFP to interact with p53. Not only wild-type EFP but also its catalytically inactive mutant, of which the active site Cys13 and Cys16 residues were replaced by serine $(\mathrm{C} 13 / 16 \mathrm{~S})$, could interact with p53 (Fig. 5a), indicating that the catalytic activity of EFP is not required for its interaction with p53. In addition, overexpression of EFP, but not its inactive form (C13/16S), markedly increased p53 ISGylation (Fig. 5b). Furthermore, knockdown of EFP by shEFP prevented DNA damage-induced p53 ISGylation (Fig. 5c), indicating that EFP serves as an E3 ligase of p53. In contrast, HERC5 was unable to interact with p53 (Fig. 5d). Moreover, treatment with DNA-damaging agents did not show any effect on HERC5 expression in both $p 53^{+/+}$and $p 53^{-/-}$HCT116 cells, unlike that on EFP expression (Fig. 5e). In addition, knockdown of HERC5 showed little or no effect on ultraviolet-induced p53 ISGylation in $p 53^{+/+}$HCT116 cells (Fig. 5f). These results indicate that neither DNA damage nor p53 influences the expression of HERC5.

To map the regions for the interaction between p53 and EFP, we first examined the ability of p53 deletions (P $\Delta 1$ to $\mathrm{P} \Delta 4)$ to interact with EFP. $\mathrm{P} \Delta 1$ (amino acid 1-300) and $\mathrm{P} \Delta 3$ (201-393), but not P $\Delta 2$ (1-200) and P $\Delta 4$ (301-393), could interact with EFP, indicating that EFP-binding site is present in the middle region of p53 (201-300) (Supplementary Fig. 10a). Various deletions of EFP (termed E $\Delta 1$ to E $\Delta 4$ ) were also generated and tested for their ability to bind p53. E $\Delta 1$ (1-438) and $\mathrm{E} \Delta 3$ (218-630) were capable of binding to p53, whereas E $\Delta 2$ (1-217) and $\mathrm{E} \Delta 4$ (439-630) could not (Supplementary Fig. 10b). These results indicate that p53-binding site lies in the middle region of EFP (218-438).

ISGylation of p53 promotes its transactivity. Of note was the finding that knockdown of ISG15 or EFP results in a significant reduction in p53 expression (see Figs $4 \mathrm{~b}$ and 5c), raising a possibility that p53 ISGylation might be involved in the control of its transactivity, in addition its stability, and thereby in the expression of its target genes (including its own). To test this possibility, p53 and its ISGylation-defective $2 \mathrm{KR}$ mutant were expressed in p53-null $\mathrm{H} 1299$ cells that had been transfected with p53-responsive reporter vectors, including PG13-Luc, p21-Luc and $B A X-L u c$. The $2 \mathrm{KR}$ mutation caused a marked decrease in ultraviolet-induced p53 transactivity (Fig. 6a). Similar results were obtained when doxorubicin was treated to cells (Supplementary Fig. 11). Consistently, prevention of p53 ISGylation by knockdown of ISG15 or EFP also dramatically reduced the p53 activity and this reduction could be reversed by co-expression of shRNA-insensitive ISG15 or EFP (Fig. 6b). Immunoblot data for cells used in Fig. 6a,b were shown in Supplementary Fig. 12a,b, respectively. These results indicate that p53 ISGylation promotes the expression of its target genes as well as of its own gene.

To test a possibility whether ISGylation influences Chk1 phosphorylation and thereby promotes the expression of ISG15conjugating system, $p 53^{+}+$HCT116 cells transfected with shISG15 or shEFP were exposed to ultraviolet. Knockdown of ISG15 or EFP markedly reduced p53 expression, but showed little or no effect on Chk1 phosphorylation (Supplementary Fig. 13). These results indicate that p53 ISGylation positively controls the expression of ISG15-conjugating system without any influence on the activation of its upstream regulators.

In an attempt to determine the mechanism for ISGylationmediated stimulation of p53 transactivity, we examined the effect of p53 ISGylation on the binding of p53 to the promoters of its target genes. ChIP analysis revealed that the expression of ISG15conjugating system with p53 in $p 53^{-/-}$HCT116 cells markedly increases the binding of $\mathrm{p} 53$ to p53REs of its target genes, 
a

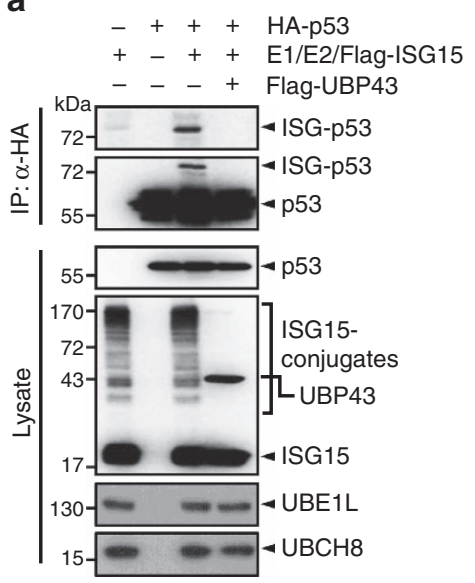

b

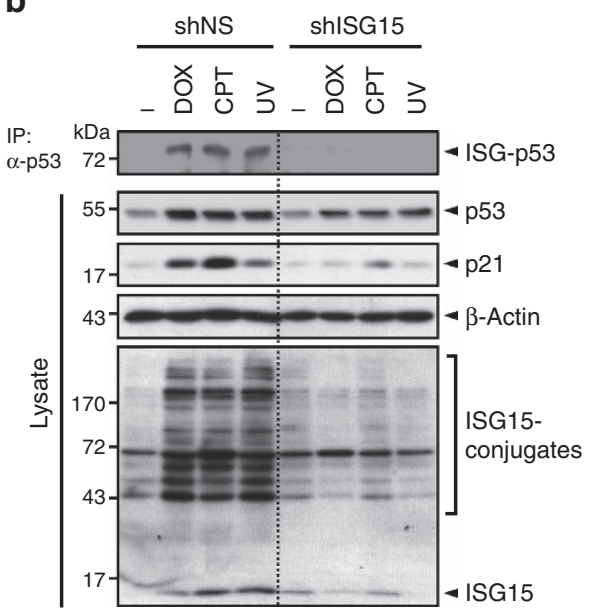

e

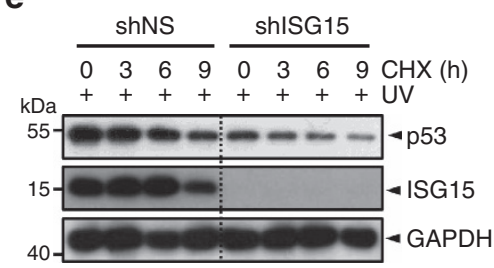

f
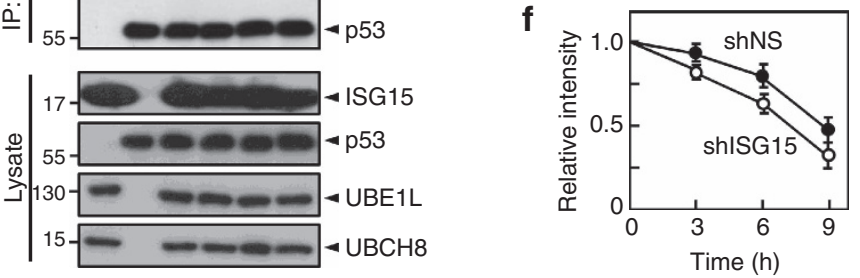

E1/E2/ISG15

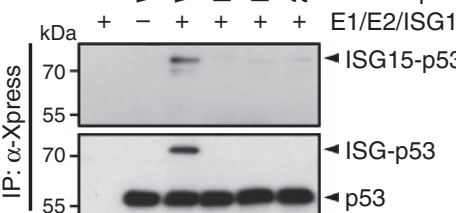

C

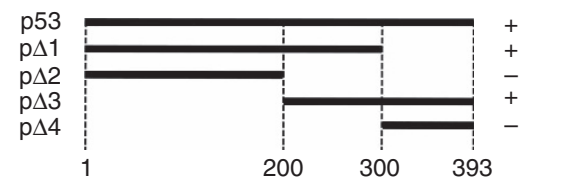

$-++-\quad-\quad-$ HisMax-p53

$-\quad-+\ldots+$ - HisMax-p $\Delta 1$

$-{ }_{-}-++-$HisMax-p $\Delta 2$

$-\quad-\quad-+-$ HisMax-p $\Delta 4$

$-\quad-\quad-\quad+$ HisMax-p $\Delta 3$

$\mathrm{kDa}+-+++++$ E1/E2/Flag-ISG15
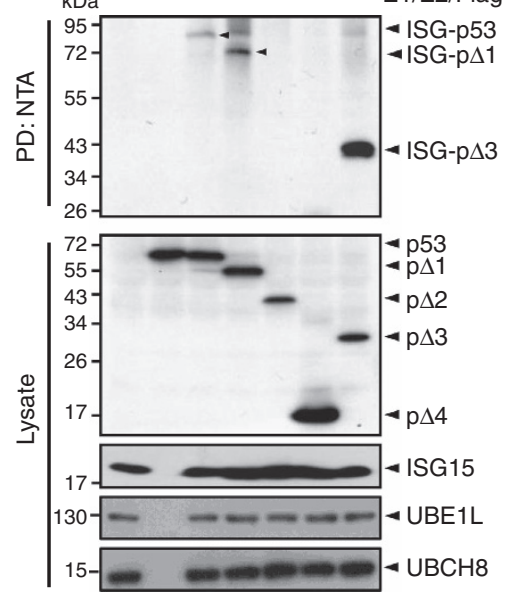

Figure 4 I DNA damage induces p53 ISGylation. (a) HA-p53 and Flag-ISG15, Myc-UBE1L (E1) and Myc-UBCH8 (E2) were expressed in HEK293T cells with or without Flag-UBP43. The cell lysates were subjected to immunoprecipitation (IP) with anti-HA antibody followed by immunoblot with anti-Flag or anti-HA antibody. They (lysates) were also directly probed with anti-HA, anti-Flag or anti-Myc antibody. (b) p53 $+/+\mathrm{HCT116}$ cells that had been transfected with shNS or shISG15 were treated with doxorubicin (DOX) or camptothecin (CPT) for $24 \mathrm{~h}$. They were also irradiated with ultraviolet (UV), and then incubated for $24 \mathrm{~h}$. The cell lysates were subjected to immunoprecipitation with anti-p53 antibody followed by immunoblot with anti-ISG15 antibody. They were also directly probed with respective antibodies. (c) Deletions of p53 (p $\Delta 1-\mathrm{p} \Delta 4$ ) were tagged with HisMax to their N-termini, and expressed in HEK293T cells with Flag-ISG15, Myc-UBE1L and Myc-UBCH8. The cell lysates were subjected to pull-down with NTA resins followed by immunoblot with anti-Flag antibody. (d) Wild-type p53 (Wt) or its K-to-R mutants were expressed in HEK293T cells with Flag-ISG15, Myc-UBE1L and MycUBCH8. The cell lysates were subjected to immunoprecipitation with anti-Xpress antibody followed by immunoblot with anti-Flag or anti-Xpress antibody. (e) HCT116 (p53 ${ }^{+/+}$) cells were transfected with shNS or shISG15. After exposure to ultraviolet, the cells were subjected to incubation with 0.2 mg ml 1 cycloheximide ( $\mathrm{CHX}$ ) for increasing periods followed by immunoblot analysis. (f) Experiments in e were repeated and the band intensities were scanned by using a densitometer and normalized by those of GAPDH. The normalized densities seen at ' 0 ' time points were expressed as 1.0 and the others were expressed as its relative values. Error bar, \pm s.d. $(n=3)$.

including $p 21, M D M 2, B A X$ and ISG15, and this increase could be abrogated by co-expression of UBP43 (Fig. 6c). On the other hand, the expression of ISG15-conjugating system showed little or no effect on the binding of ISGylation-deficient $2 \mathrm{KR}$ mutant to p53REs of its target genes (Fig. 6d). Furthermore, knockdown of ISG15 dramatically reduced ultraviolet-induced binding of p53 to the promoter regions but this effect could be reversed upon complementation of a shRNA-insensitive ISG15 (Fig. 6e). Similar results were obtained when experiments in Fig. $6 \mathrm{c}-\mathrm{e}$ were repeated and the extracted DNAs were subjected to quantitative PCR analysis (Supplementary Fig. 14). These results indicate that p53 ISGylation plays a crucial role in the promotion of $\mathrm{p} 53$ binding to the promoters of its target genes under DNA damage conditions.

Acetylation of p53 has been shown to strongly increase its affinity of $p 53 R E^{39,40}$. In addition, it has been shown that p53 phosphorylation increases its binding to p300 acetyl-transferase $^{41,42}$. To determine whether p53 ISGylation influences its phosphorylation and acetylation, H1299 cells expressing wildtype p53 or its $2 \mathrm{KR}$ mutant were exposed to ultraviolet. Immunoblot analysis revealed that the $2 \mathrm{KR}$ mutation almost completely abrogates ultraviolet-induced acetylation of p53 (Supplementary Fig. 15a,b). It also significantly inhibited p53 phosphorylation. These results indicate that p53 ISGylation promotes its phosphorylation and acetylation and, in turn, its ability to bind to $p 53 R E$. These results also raised a possibility that under DNA damage conditions, p53 might be ISGylated, initially by the basal ISG15 and its conjugating system for early activation of $\mathrm{p} 53$ by phosphorylation and acetylation and then by belatedly induced ISG15-conjugating system for further potentiation of p53 transactivity. To test this possibility, we examined whether p53 ISGylation occurs before its phosphorylation and acetylation 
a

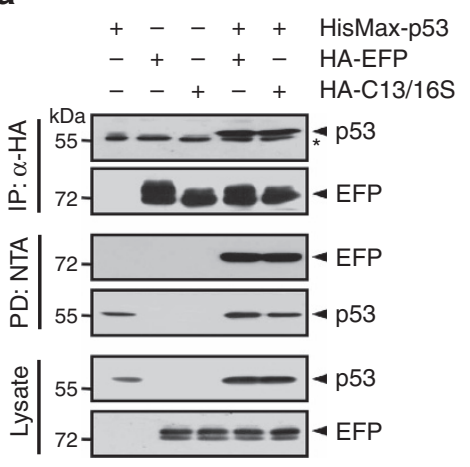

b

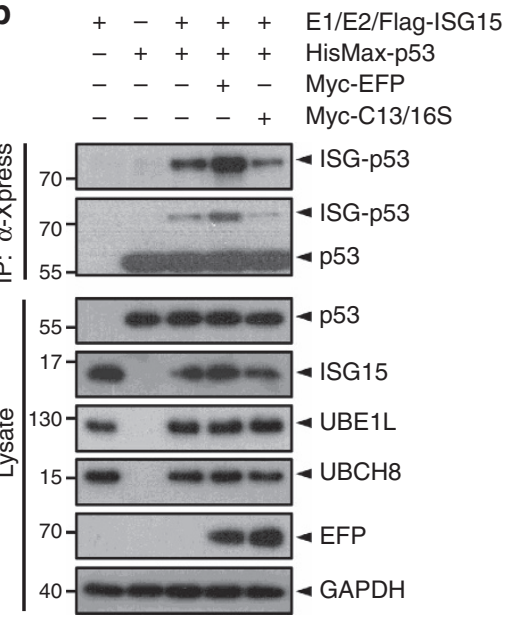

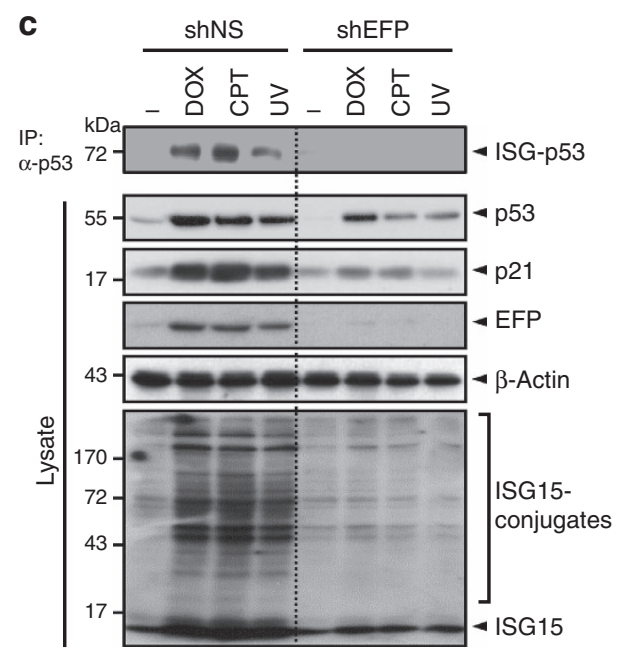

$\mathbf{f}$

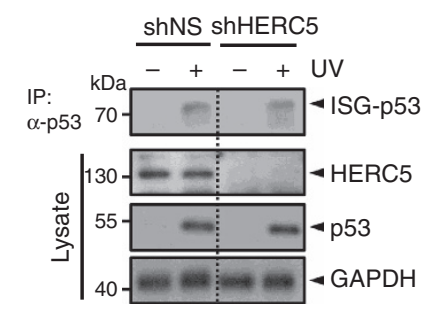

d

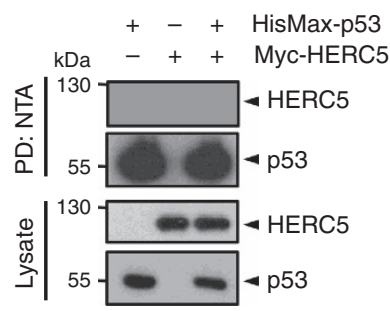

e

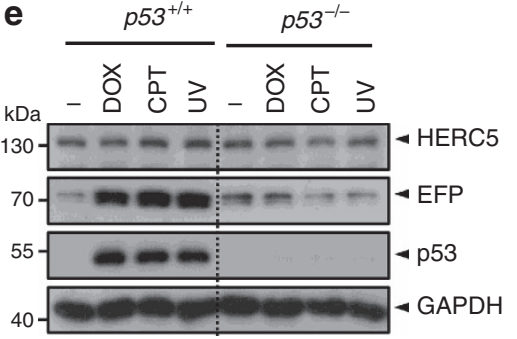

Figure 5 | EFP serves as a p53-specific ISG15 E3 ligase. (a) HA-EFP or its inactive C13/16S mutant was expressed in HEK293T cells with HisMax-p53. The cell lysates were subjected to immunoprecipitation with anti-HA antibody or pull-down with NTA resins followed by immunoblot with anti-Xpress or antiHA antibody. The asterisk indicates lgG heavy chain. (b) HisMax-p53, Flag-ISG15, Myc-UBE1L (E1) and Myc-UBCH8 (E2) were expressed in HEK293T cells with Myc-tagged EFP or its C13/16S mutant. The cell lysates were subjected to immunoprecipitation with anti-Xpress antibody followed by immunoblot anti-Flag or anti-Xpress antibody. (c) $p 53^{+/+}$HCT116 cells that had been transfected with shNS or shEFP were treated with doxorubicin or camptothecin for $24 \mathrm{~h}$. They were also treated with ultraviolet (UV), and then incubated for $24 \mathrm{~h}$. The cell lysates were subjected to immunoprecipitation with anti-p53 antibody followed by immunoblot with anti-ISG15 antibody. (d) Myc-HERC5 was expressed in HEK293T cells with HisMax-p53. The cell lysates were subjected to pull-down with NTA resins followed by immunoblot with anti-Myc or anti-Xpress antibody. (e) After treatment with DNA-damaging agents, the HCT116 cells were subjected to incubation for $24 \mathrm{~h}$ followed by immunoblot with anti-HERC5, anti-EFP or anti-p53 antibody. (f) p53+/ + HCT116 cells expressing shNS or shHERC5 were exposed to ultraviolet. After incubation for $24 \mathrm{~h}$, the cells were subjected to immunoprecipitation with anti-p53 followed by immunoblot with anti-ISG15 antibody.

immediately after ultraviolet treatment. The levels of $\mathrm{p} 53$, phospho-p53 and acetylated p53 gradually increased in order, whereas ISGylated p53 could not be detected at least for $3 \mathrm{~h}$ after ultraviolet treatment (Supplementary Fig. 15c). These results indicate that phosphorylation and acetylation of $\mathrm{p} 53$ precede its ISGylation for induction of ISG15-conjugating system as well as of other downstream targets.

p53 ISGylation suppresses cell growth and tumorigenesis. To determine whether p53 ISGylation influences its ability to inhibit cell growth, p53 or its $2 \mathrm{KR}$ mutant was expressed in $p 53^{-/-}$ HCT116 cells that had been transfected with shNS or shISG15. Doxorubicin treatment led to a marked decrease in the survival of p53-expressing cells, but showed much less effect on the survival of 2KR-expressing cells, and these inhibitory effects on cell survival could be abrogated by ISG15 knockdown (Fig. 7a). Furthermore, the clonogenic assays revealed that $2 \mathrm{KR}$-expressing cells are much less sensitive to the drug-mediated inhibition of colony formation than wild-type p53-expressing cells and these effects could be abolished by ISG15 knockdown (Fig. 7b,c). Consistently, knockdown of ISG15 markedly reduced doxorubicin-mediated inhibition of colony formation of $p 53^{+/+}$ HCT116 cells and this reduction could be prevented upon co-expression of shRNA-insensitive ISG15 (Fig. 7d). However, ISG15 knockdown regardless of co-expression of shRNA-insensitive ISG15 showed little or no effect on colony formation by p53 ${ }^{-/-}$HCT116 cells. Immunoblot data for cells used in Fig. 7a,b,d were shown in Supplementary Fig. 16a-c, respectively. Similar results were obtained when cells were treated with camptothecin or ultraviolet in place of doxorubicin. We next examined whether p53 ISGylation promotes apoptosis under DNA damage conditions. $p 53^{-/-}$HCT116 cells expressing wildtype p53 or its $2 \mathrm{KR}$ mutant were treated with different concentrations of doxorubicin. TUNEL assay revealed that apoptosis is markedly reduced in cells expressing $2 \mathrm{KR}$ mutant compared with those expressing wild-type p53 (Supplementary Fig. 17). Collectively, these results indicate that DNA damage-induced ISGylation of p53 promotes cell growth inhibition and apoptosis by increasing its transactivity.

To determine whether ISGylation of p53 could consequently promote its tumour suppressive function, an in vivo tumorigenesis assay was performed by injecting BALB/c nude mice with $p 53^{+/+}$HCT116 cells stably expressing shNS or shISG15 and $p 53^{-/-}$HCT116 cells stably expressing wild-type p53 and its $2 \mathrm{KR}$ mutant. Without doxorubicin treatment, all the mice developed large tumours (Fig. 7e and Supplementary Fig. 18). On the drug treatment, tumour volumes were dramatically 
a

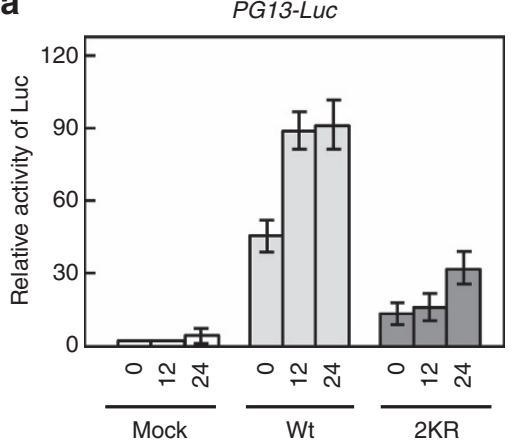

b

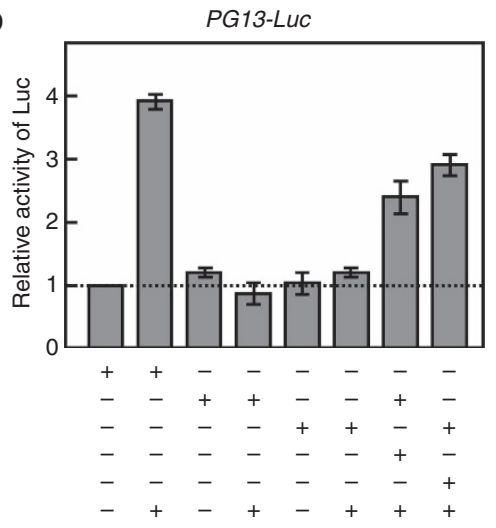

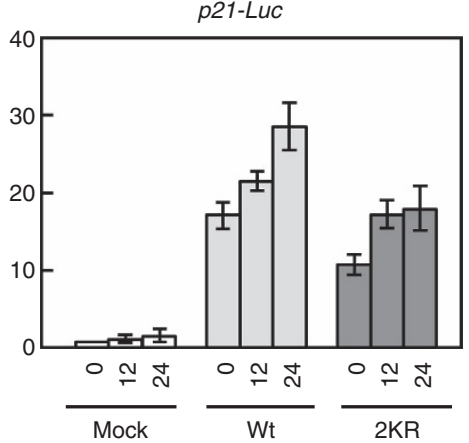
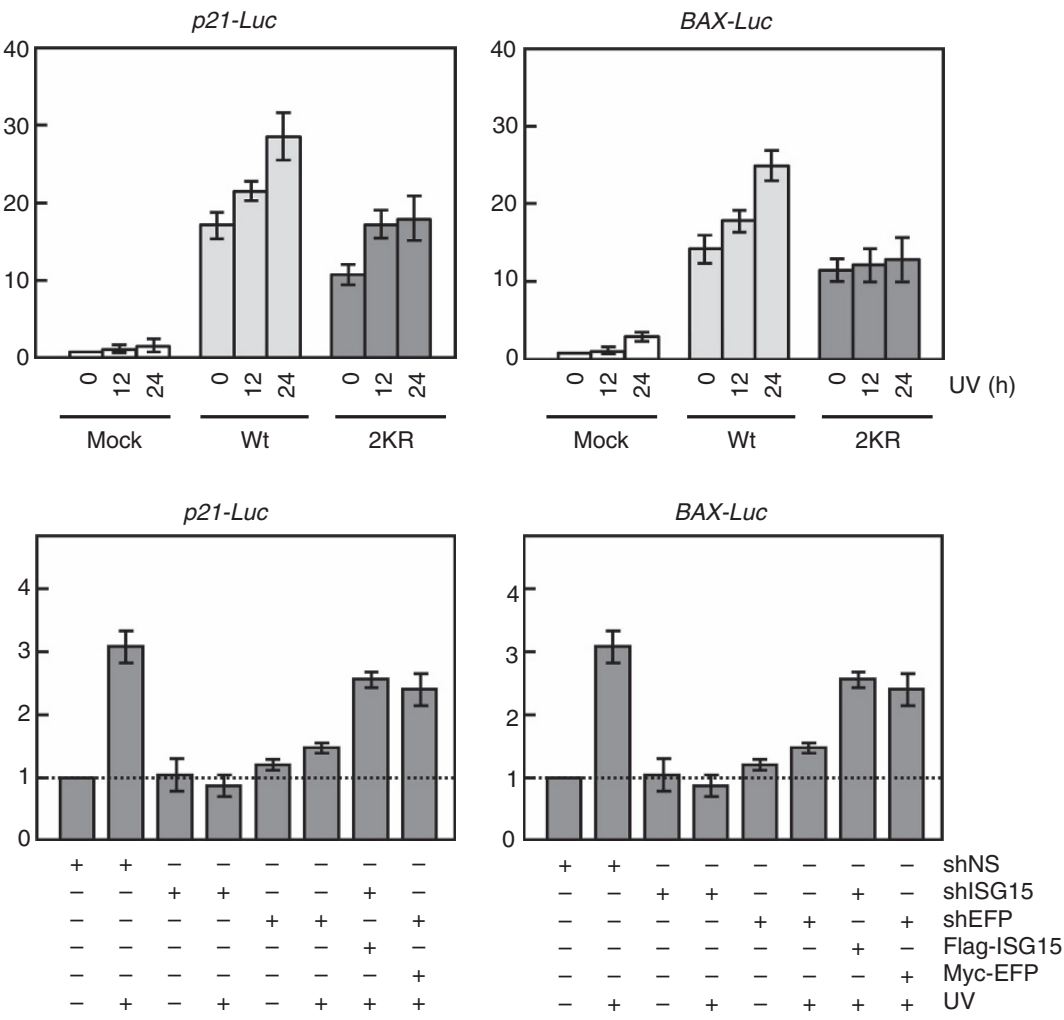

C
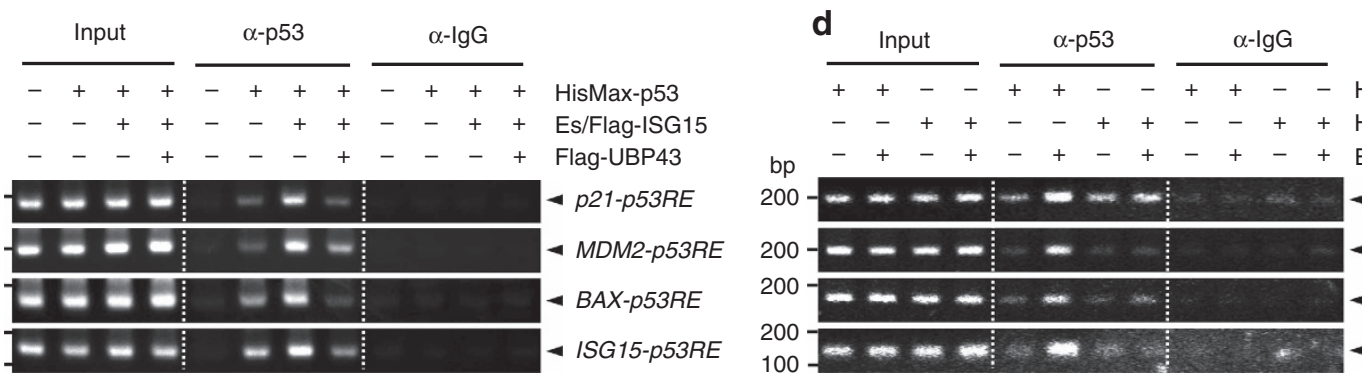

HisMax-p53

$--_{-}+-c_{+}+-++$Es/Flag-ISG15

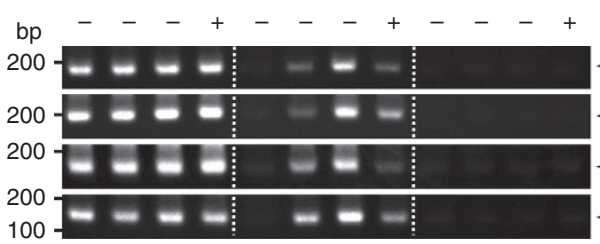

p21-p53RE

MDM2-p53RE

- BAX-p53RE

ISG15-p53RE

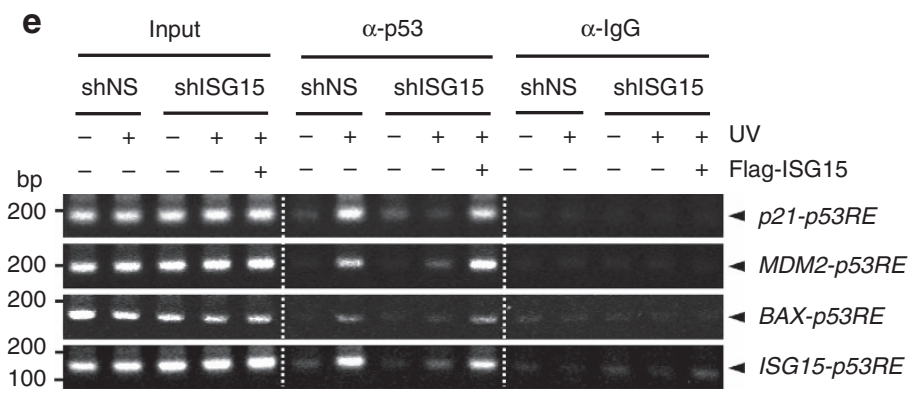

Figure 6 | ISGylation promotes p53 transactivity. (a) Wild-type p53 (Wt), its 2KR mutant, and an empty vector (Mock) were expressed in H1299 cells with PG13-Luc, p21-Luc and BAX-Luc. After exposure to ultraviolet (UV), they were incubated for various periods, and subjected to assay for the luciferase activity. Transfection efficiency was normalized by using $\beta$-galactosidase constructs. The luciferase activity seen without any treatment (that is, ' 0 ' time in Mock) was expressed as 1.0 and the others were as its relative values. Error bar, \pm s.d. $(n=3)$. (b) shNS, shISG15 or shEFP was expressed in $p 53^{+} /+$ HCT116 cells with and without shRNA-insensitive Flag-ISG15 or Myc-EFP. After exposure to ultraviolet, the cells were incubated for $24 \mathrm{~h}$ and subjected to the assay for the luciferase activity as in a. Error bar, \pm s.d. $(n=3)$. (c) HisMax-p53 and ISG15-conjugating system (Es/Flag-ISG15) were expressed in p53-/- HCT116 cells with and without Flag-UBP43. The cell lysates were subjected to ChIP assay using anti-p53 antibody or anti-mouse IgG. Bound DNAs were subjected to PCR using the probes for p53REs of CDKN1, MDM2, BAX and ISG15. (d) HisMax-p53 and HisMax-2KR were expressed in p53-/HCT116 cells with or without ISG15-conjugating system. The cell lysates were subjected to ChIP as in c. (e) shNS or shISG15 were expressed in p53+/+ HCT116 cells with and without shRNA-insensitive Flag-ISG15. After exposure to ultraviolet, the cells were incubated for $24 \mathrm{~h}$, and then subjected to ChIP as in $\mathbf{c}$. Note that shISG15 was directed to a $5^{\prime}$-UTR region. $\mathbf{a}, \mathbf{b}$ and $\mathbf{e}$, similar results were obtained when the cells were treated with doxorubicin or camptothecin in place of ultraviolet. 
a
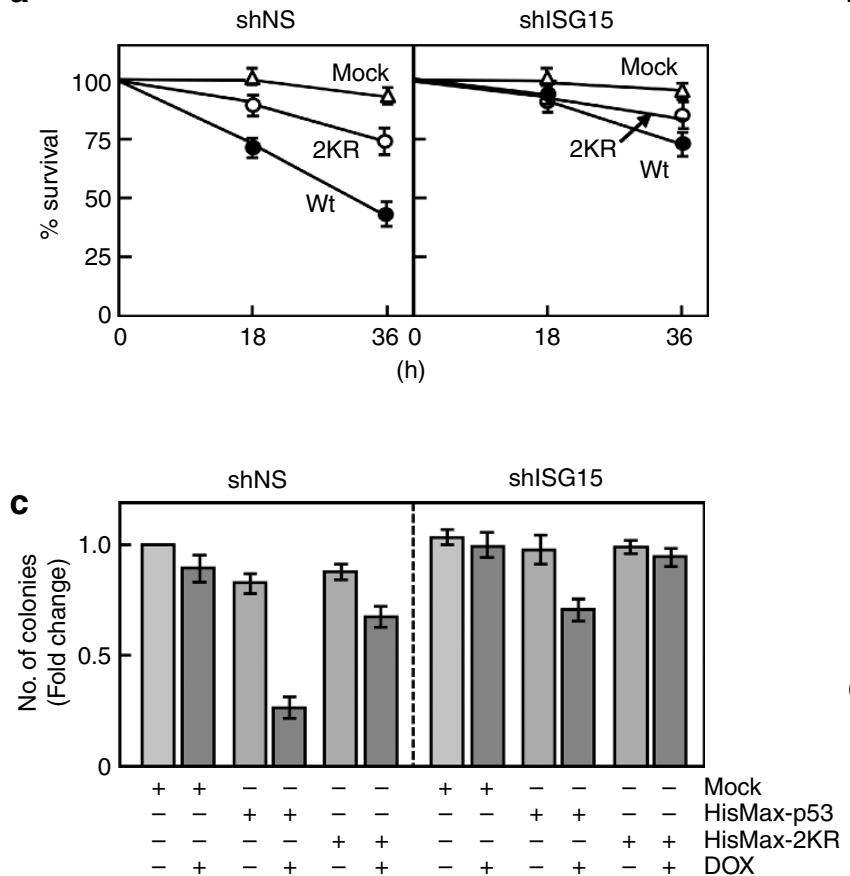

e
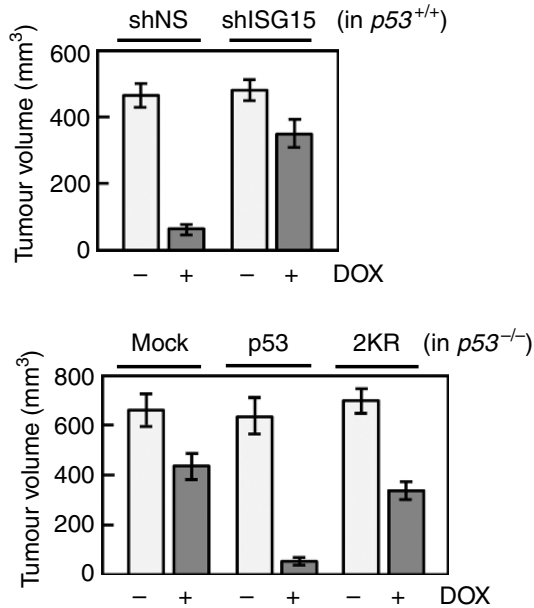

b

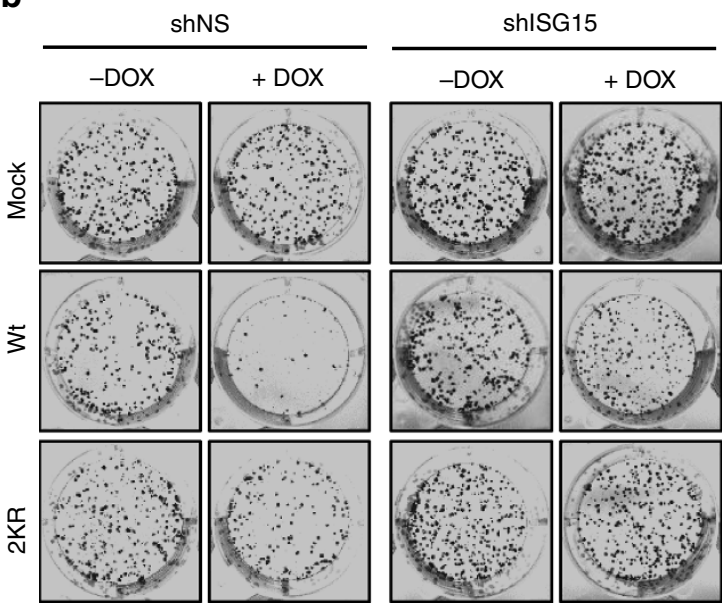

d

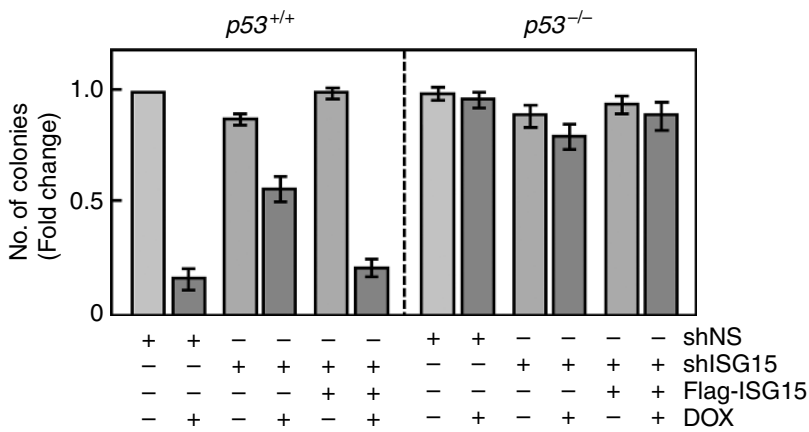

f

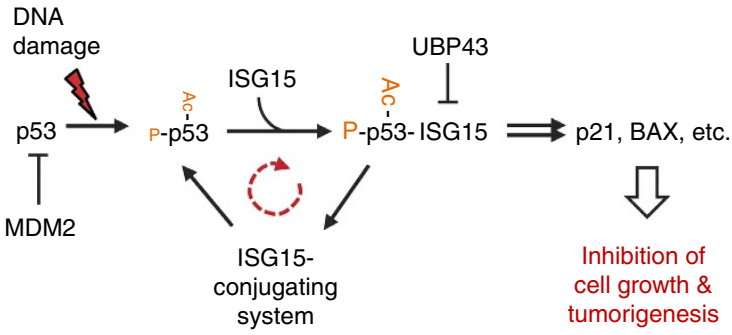

Figure 7 | p53 ISGylation suppresses cell growth and tumour development. (a) p53-/- HCT116 cells expressing wild-type p53 (Wt), its $2 \mathrm{KR}$ mutant, or an empty vector (Mock) were transfected with shNS or shISG15. After treatment with doxorubicin for increasing periods, viable cells were counted by staining with trypan blue exclusion. Error bar, \pm s.d. $(n=3)$. (b) $p 53^{-/-}$HCT116 cells prepared as in a were incubated with or without doxorubicin for 10 days. The colonies were then stained with crystal violet. (c) Experiments were repeated as in $\mathbf{b}$ and the colonies were counted. Error bar, $\pm \mathrm{s}$.d. $(n=3)$ (d) shNS or shISG15 were expressed in both $p 53^{+/+}$and $p 53^{-/-}$HCT116 cells with and without shRNA-insensitive Flag-ISG15. After treatment with doxorubicin, experiments were then performed as in b. Error bar, \pm s.d. $(n=3)$. (e) $p 53^{+/+}$HCT116 cells expressing shNS or shISG15 (top) and $p 53^{-/-}$ HCT116 cells expressing wild-type p53 or its 2KR mutant (bottom) were injected to BALB/c nude mice. 'Mock' in the bottom panel indicates the cells transfected with an empty vector. After treatment with PBS or doxorubicin, tumour volumes were determined. Error bar, \pm s.d. ( $n=5$ ). (f) A model for positive feedback regulation of p53 transactivity by ISGylation under DNA damage conditions. 'Ac' and 'P' indicate acetylated and phosphorylated, respectively.

reduced in mice injected with $p 53^{+/+}$cells expressing shNS and $p 53^{-/-}$cells expressing ectopic p53. Consistently, immunoblot analysis of these tumour tissues showed a marked increase in the level of apoptotic BAX protein. In contrast, tumours with appreciable sizes were developed in mice injected with $p 53^{+/+}$cells expressing shISG15 and $p 53^{-/-}$cells expressing $2 \mathrm{KR}$ mutant, even when doxorubicin was treated, indicating the involvement of p53 ISGylation in tumour suppression. Notably, however, the size of tumour developed in mice injected with $p 53^{-/-}$cells expressing $2 \mathrm{KR}$ mutant is smaller than that in mice $p 53^{-/-}$cells transfected with an empty vector (Mock) under doxorubicin-treated conditions, indicating that $2 \mathrm{KR}$ is still capable of suppressing tumour development by inducing p53 phosphorylation and BAX expression, although much less efficiently than wild-type p53. Collectively, these results indicate that ISGylation of p53 plays a crucial role in promotion of its tumour-suppressive function. 
UBP43 represses expression of ISG15-conjugating system. We have recently shown that ISGylation of PCNA terminates ultraviolet-induced, error-prone translesion DNA synthesis and that the ISGylation process is reversed by UBP43, whose expression is induced at the later periods, for regaining the function of PCNA as a processive factor in normal replication ${ }^{33}$. To determine whether UBP43 expression is also induced at the later periods under different DNA damage conditions, $p 53^{+/+}$HCT116 cells expressing shNS or shISG15 were treated with doxorubicin for increasing periods. Immunoblot analysis revealed that in shNSexpressing cells, the levels of p53, p-p53, MDM2, p21 and BAX gradually increased until $24-36 \mathrm{~h}$ after doxorubicin treatment, and remained elevated (or began to fall) thereafter without further increase (Supplementary Fig. 19, left panels). The levels of ISG15-conjugating system also increased until 24-36 h after the drug treatment, but declined thereafter, concomitantly with a marked increase in the level of UBP43. These results strongly suggest that UBP43 deISGylates p53 at the later period of doxorubicin treatment and thereby downregulates the expression of ISG15-conjugating system for prevention of prolonged promotion of $\mathrm{p} 53$ transactivity.

Upon depletion of ISG15, the expression of p53, p-p53, MDM2, p21 and BAX was markedly reduced and delayed, although their levels gradually increased at the later periods after doxorubicin treatment (Supplementary Fig. 19, right panels). In addition, ISG15 knockdown abrogated doxorubicin-induced riseand-fall of the expression of ISG15-conjugating system, confirming that p53 ISGylation promotes the expression of its downstream targets. Interestingly, however, ISG15 knockdown showed little or no effect on the expression time and level of UBP43, indicating that the expression of UBP43 is not associated with p53 ISGylation. However, it remains unknown how the belated expression of UBP43 is regulated under DNA damage conditions.

\section{Discussion}

In the present study, we showed that the promoters of the ISG15, $U B E 1 L$, UBCH8 and EFP genes have p53REs for their p53-mediated expression, independent of type-I IFNs. We further demonstrated that p53 serves as a target for conjugation by ISG15 and this modification dramatically stimulates the binding of p53 to $p 53 R E s$ by promoting its phosphorylation and acetylation, indicating a positive feedback regulatory circuit operates for potentiation of p53 transactivity. On the basis of this finding, we propose a model for the role of DNA damage-induced ISGylation in the control of p53 transactivity and, in turn, in inhibition of cell and tumour growth (Fig. 7f). Under normal conditions, the cellular level of p53 is kept low by MDM2-mediated ubiquitination and proteasomal degradation. On exposure to DNAdamaging agents, such as doxorubicin, camptothecin and ultraviolet, the p53 level gets elevated and activated for expression of its downstream targets, including ISG15-conjugating system, and thereby for ISGylation of p53 (as well as of other substrates), albeit initially to a low level. This ISGylation dramatically stimulates phosphorylation and acetylation of p53, which promotes its binding to $p 53 R E$ for more efficient and belated expression of p53 itself and its downstream targets, including p21, BAX and ISG15-conjugating system. Thus, it appears that the sequential phosphorylation, acetylation and ISGylation of p53 forms a cycle with the expression of ISG15-conjugating system for amplified expression of p53 downstream targets. Collectively, the positive feedback regulation of p53 transactivity by ISG15 modification seems to play a crucial role in the expression of its target genes involved in cell growth inhibition and, in turn, in suppression of tumour development under DNA damage conditions. Interestingly, however, the expression of UBP43 is induced at the later periods after treatment with DNA-damaging agents. This increase in UBP43 expression should lead to p53 de-ISGylation for termination of positive feedback control of p53 transactivity, particularly in cells that had already committed to apoptotic cell death as well as in residual cells that survived after DNA repair.

Of note was the finding that ISGylation of p53 leads to a marked increase in its ability to bind p53REs. Previously, it was shown that p53 phosphorylation increases its binding to p300 acetyl-transferase and p53 acetylation dramatically promotes its binding to $p 53-R E^{39-42}$. In this study, we found that ultravioletinduced p53 ISGylation promotes its phosphorylation and acetylation. Thus, it appears likely that ISG15 molecules conjugated to p53 serve as molecular scaffolds that recruit DNA damage-activated protein kinase(s) and acetyl-transferases(s), such as p300, for phosphorylation and acetylation of p53 and thereby for elevating its affinity to p53REs.

Recently, Huang et al. ${ }^{16}$ have shown that p53 can be ISGylated at multiple unknown sites by overexpression of HERC5, but not by EFP. They also showed that misfolded p53 is preferentially ISGylated for proteasomal degradation and that ISG15 depletion leads to the accumulation of misfolded p53, which then acts dominant negatively on $\mathrm{p} 53$ function, such as on apoptosis. This HERC5-mediated ISGylation of p53 is in contrast with our finding that p53 interacts with EFP, but not HERC5, even under overexpression conditions. Moreover, knockdown of EFP, but not HERC5, was found to prevent DNA damage-induced p53 ISGylation (Fig. 5c, f). However, it has been shown that HERC5 is physically associated with polyribosomes, and can modify a wide range of newly synthesized proteins by ISG15 in a co-translational manner ${ }^{43}$. Therefore, it appears possible that polyribosomeassociated HERC5 ISGylates newly synthesized, unstructured p53 protein without strong interaction with the E3 ligase.

Previously, we have shown that doxorubicin can induce the expression of ISG15, UBE1L and UBCH8, leading to ISGylation of $\triangle \mathrm{Np} 63 \alpha$, in HNSCC013 and HCC1937 cells, despite the fact that these cells express mutated nonfunctional p53 (refs 44,45). Thus, it appears that certain cell types are capable of inducing ISG15-conjugating system independently of p53 under DNA damage conditions. It has been reported that $\Delta \mathrm{Np} 63 \alpha$, lacking the amino (N)-terminal transactivation domain, has a second transactivation domain, and therefore can regulate expression of distinct subset of genes ${ }^{46-48}$, although it is better known as p53 inhibitor, owing to the presence of the carboxy (C)-terminal transcriptional inhibition domain ${ }^{49,50}$. Alternatively, therefore, $\Delta \mathrm{Np} 63 \alpha$ itself might induce ISG15-conjugating system for its own ISGylation.

Protein ISGylation has been implicated in both tumour development and tumour suppression ${ }^{51}$. The level of ISG15 is elevated in many human primary cancers, such as in tumours of bladder, breast, endometrium and prostate ${ }^{52-56}$. Recently, knockout of ISG15 was shown to suppress K-ras-induced lung tumour as well as V-Src-mediated tumour formation in mice ${ }^{57}$. In addition, UBP43-deficient mice have been shown to develop early spontaneous leiomyosarcoma tumours with occasional stabilization of p53 (ref. 58). Conversely, it has also been reported that repression of UBP43 shows antineoplastic effects in lung and kidney cancers and in acute promyelocytic leukemia $^{59-61}$. In addition, UBE1L, the ISG15-activating E1 enzyme, has been shown to play a role in suppression of lung cancer and leukemia by promoting ISGylation of cyclin D1 and PML-RAR $\alpha$ fusion protein, respectively ${ }^{62-66}$. Moreover, DNA-damaging agents, such as doxorubicin, were shown to induce ISGylation and caspase-2-mediated cleavage of ISGylated $\Delta \mathrm{Np} 63 \alpha$, an anti-apoptotic and mitogenic oncoprotein, implicating the role of the ISG15 system in suppression of 
$\Delta \mathrm{Np} 63 \alpha$-mediated tumorigenesis ${ }^{32}$. It has also been shown that ISGylation of PCNA plays a critical role in termination of ultraviolet-induced error-prone translesion synthesis and thus in the maintenance of genome stability for preventing DNA damage-induced tumorigenesis ${ }^{33}$. Finally, in this study, we have shown that DNA damage-induced p53 ISGylation inhibits cell growth and tumour development by promoting its transactivity. Thus, it appears that protein ISGylation plays a crucial role in positive regulation of DNA damage response, which in turn suppresses DNA damage-induced tumorigenesis.

\section{Methods}

Plasmids and antibodies. cDNAs for human ISG15 and UBCH8 and mouse UBP43 were subcloned into pFlag-CMV10 vector and/or pcDNA3-Myc vector ${ }^{32}$. Human p53 cDNAs were obtained from Korean human gene bank. p53, its deletion constructs, and K-to-R mutants were subcloned into pcDNA3-HA vector and pcDNA-HisMax vector (Invitrogen ${ }^{67}$. Site-directed mutagenesis was performed as recommended by the manufacturer's instructions (Stratagene).

Antibodies used were mouse monoclonal anti-Xpress (Catalogue number P/N 46-0528, Invitrogen), mouse monoclonal anti-Flag M2 (F3165, Sigma), mouse monoclonal anti-Myc (9E10, Santa Cruz), mouse monoclonal anti-p53 (DO-1, Santa Cruz), rabbit polyclonal anti-p21 (C-19, Santa Cruz), mouse monoclonal anti- $\beta$-actin (C4, Santa Cruz), rabbit anti-phospho-p53 (9284S, Cell Signaling) and rabbit anti-acetyl-p53 (2525S, Cell Signaling). Polyclonal anti-ISG15 antibody was generated by injecting purified ISG15 protein to rabbit ${ }^{32}$. shRNA were purchased from Open Biosystems. Target sequences of shRNAs for ISG15 and EFP are: 5'-CTGAGCATCCTGGTGAGGAAT- $3^{\prime}$ for ISG15; $5^{\prime}$-GAACTCATCTTTGCC AGTA- $3^{\prime}$ (5'-UTR (untranslated region) for ISG15; $5^{\prime}$-GAGTGAGATCCAGAC CTTGAA-3' for EFP; $5^{\prime}$-GCAGAACTCTCCTTGGATA-3' (3'-UTR) for EFP. All antibodies were diluted 1:1,000 with PBS containing $0.1 \%$ Triton X-100 and 3\% BSA, except anti- $\beta$-actin, which was diluted 1:2,500.

Immunoprecipitation and pull-down assay. For immunoprecipitation, cell lysates were prepared in buffer-A consisting of $50 \mathrm{mM}$ Tris- $\mathrm{HCl}(\mathrm{pH} 7.4), 150 \mathrm{mM}$ $\mathrm{NaCl}, 1 \mathrm{mM}$ EDTA, $1 \mathrm{mM}$ NEM, $1 \mathrm{mM}$ sodium orthovanadate, $1 \mathrm{mM} \mathrm{NaF}, 1 \mathrm{mM}$ PMSF, $0.2 \%$ (v/v) Triton X-100 and $1 \mathrm{X}$ protease inhibitor cocktail (Roche). The cell lysates were incubated with appropriate antibodies for $1 \mathrm{~h}$ at $4{ }^{\circ} \mathrm{C}$ and then with protein A-conjugated Sepharose for the next $1 \mathrm{~h}$. For $\mathrm{Ni}^{2+}$-NTA agarose (NTA: Qiagen) pull-down assay, the cell lysates were prepared in buffer-A containing $10 \mathrm{mM}$ imidazole. After incubating cell lysates with NTA resin for $1 \mathrm{~h}$ at $4{ }^{\circ} \mathrm{C}$, the samples were precipitated, washed three times with buffer-A containing $20 \mathrm{mM}$ imidazole and subjected to SDS-polyacrylamide gel electrophoresis followed by immunoblot analysis. Supplementary Fig. 20 shows the full-blots of the data obtained from SDS-polyacrylamide gel electrophoresis.

Cell culture and transfection. HEK293T, A549 and H1299 cells (from ATCC) were cultured at $37^{\circ} \mathrm{C}$ in $5 \% \mathrm{CO}_{2}$ atmosphere in DMEM supplemented with $10 \%$ FBS, $2 \mathrm{mM}$ L-glutamine and $25 \mathrm{units}^{-1}$ of penicillin and streptomycin. HCT116 cells were cultured as above, but using RPMI1640 medium in place of DMEM. Transfections of plasmids were performed using Metafectene (Biontex) and JetPEI (Polyplus) according to the manufacturer's instructions. All the cell lines were regularly tested for mycoplasma contamination.

ISGylation assays. ISG15-conjugating system was overexpressed in HEK293T cells with HA- or HisMax-tagged p53. The cell lysates were prepared in buffer-A. The samples were incubated with appropriate antibodies for $2 \mathrm{~h}$ at $4{ }^{\circ} \mathrm{C}$ and then with protein A-conjugated Sepharose for the next $1 \mathrm{~h}$. The precipitates were washed and subjected to immunoblot analysis.

Luciferase assay. H1299 cells transfected with pcDNA- $\beta$-gal and PG13-Luc, p21$L u c$ or $B A X-L u c$ were incubated for $24 \mathrm{~h}$. After treatment with ultraviolet, the cell lysates were subjected to assay for the luciferase activity (Promega) as recommended by the manufacturer. Transfection efficiency was normalized by using $\beta$-galactosidase as a control.

Cell growth and clonogenic assays. For cell growth assay, $p 53^{-/-}$HCT116 cells $\left(3.0 \times 10^{5}\right)$ were cultured in triplicates in $60 \mathrm{~mm}$ plates for $24 \mathrm{~h}$. The cells were then treated with $0.1 \mu \mathrm{M}$ doxorubicin for various periods before harvesting. Viable cells were counted following trypan blue staining. For clonogenic assay ${ }^{68}, p 53^{-/-}$ HCT116 cells that stably express either HisMax-tagged wild-type p53 or its $2 \mathrm{KR}$ mutant were plated in six-well plates at 500 cells in $2 \mathrm{ml}$ of RPMI1640 medium per well. After incubation for $24 \mathrm{~h}$, the cells were treated with $0.1 \mu \mathrm{M}$ doxorubicin and further incubated for the next 10 days. The colonies developed were washed twice with PBS, fixed and stained with crystal violet for $20 \mathrm{~min}$, washed twice with PBS and then counted.
ChIP assay. HCT116 cells that had been treated with ultraviolet or doxorubicin were quickly washed with PBS, crosslinked in $1 \%$ formaldehyde for $10 \mathrm{~min}$, collected by $100 \mathrm{mM}$ Tris- $\mathrm{HCl}(\mathrm{pH} 9.4)$ containing $10 \mathrm{mM}$ DTT and incubated for $15 \mathrm{~min}$ at $30^{\circ} \mathrm{C}$. For chromatin fragmentation, the cells were sonicated in $50 \mathrm{mM}$ Tris-HCl (pH 8.1) containing 1\% SDS, $10 \mathrm{mM}$ EDTA, 1X protease inhibitor cocktail. Proteins were immunoprecipitated in $20 \mathrm{mM}$ Tris- $\mathrm{HCl}(\mathrm{pH} 8.1)$ containing $1 \%$ Triton X-100, $2 \mathrm{mM}$ EDTA, $150 \mathrm{mM} \mathrm{NaCl}$ and $1 \mathrm{X}$ protease inhibitor cocktail. Crosslinking was reversed by overnight incubation at $65^{\circ} \mathrm{C}$ in $1 \%$ SDS and $0.1 \mathrm{M} \mathrm{NaHCO}_{3}$, and fragmented DNAs were purified by using QIAquick gel extraction kit (QIAGEN). Precipitated DNAs was then subjected to PCR (40 cycles) followed by electrophoresis or to quantitative PCR. The primers used for PCR analyses were listed in Supplementary Fig. 6.

Quantitative RT-PCR. mRNA abundance was detected by using an ABI Prism 7,500 system and 2X PreMix SYBR Green (Enzynomics RT500S). Primer pairs were designed to amplify 50-200 bp mRNA-specific fragments, which were then confirmed as unique products by melting curve analysis. The PCR conditions were as follows: $95^{\circ} \mathrm{C}(15 \mathrm{~min})$ and 40 cycles of $95^{\circ} \mathrm{C}(30 \mathrm{~s}), 60^{\circ} \mathrm{C}(30 \mathrm{~s})$, and $72^{\circ} \mathrm{C}$ $(30 \mathrm{~s})$. The quantity of mRNA was calculated using the DDCt method and normalized to that of GAPDH. All the reactions were performed in triplicates. The primers used were $5^{\prime}$-GGTGGACAAATGCGACGAAC-3' (forward) and 5'-ATGCTGGTGGAGGCCCTTAG-3' (reverse) for ISG15; 5' -AGGTGGCCAAG AACTTGGTT- $3^{\prime}$ (forward) and $5^{\prime}$-CACCACCTGGAAGTCCAACA-3' (reverse) for UBE1L; 5'-GGAACCTGTCCAGCGATGAT-3' (forward) and $5^{\prime}$-TCAGGTG GTAGGGAGGTTGGT-3' (reverse) for UBCH8; $5^{\prime}$-GTGACCACGGCTTTGTCATCT- $3^{\prime}$ (forward) and 5'-TAAAGTCCACCCTGAACTTATACATCAG- $3^{\prime}$ (reverse) for EFP; 5'-GGAGGTGAAGGTCGGAGTCA-3' (forward) and 5'-GACA AGCTTCCCGTTCTCAG-3' (reverse) for GAPDH.

In vivo tumorigenesis assays. $p 53^{+/+}$HCT 116 cells $\left(5 \times 10^{6}\right)$ that stably express shNS or shISG15 and $p 53^{-1-}$ HCT116 cells $\left(5 \times 10^{6}\right)$ that stably express wild-type p53 or its ISGylation-deficient KR mutant were subcutaneously injected into the upper thighs of 4 -week-old $\mathrm{BALB} / \mathrm{c}$ male nude mice. On the third day after injection, mice were intraperitoneally injected twice weekly with PBS or doxorubicin $\left(1.25 \mathrm{mg} \mathrm{kg}^{-1}\right)$. They were monitored regularly for tumour growth. Tumour volumes were calculated as $(a \times a \times b) / 2$, in which $a$ is the smallest diameter and $b$ is the largest. Finally, the mice were killed and tumours were dissected out, photographed and subjected to immunoblot analysis.

Animal study approval. All animal procedures were approved by the IACUC of Seoul National University, Korea.

Statistics. All the values were given as mean \pm s.d. Statistical comparisons were made by two-tailed Student's $t$-test. $P$ values less than 0.05 was considered statistically significant.

Data availability. The authors declare that the data supporting the findings in this study are available within the article and its Supplementary Information files as well as upon request from the authors.

\section{References}

1. Lane, D. P. Cancer. p53, guardian of the genome. Nature 358, 15-16 (1992).

2. Levine, A. J. p53, the cellular gatekeeper for growth and division. Cell 88, 323-331 (1997).

3. Green, D. R. \& Kroemer, G. Cytoplasmic functions of the tumour suppressor p53. Nature 458, 1127-1130 (2009).

4. Riley, T., Sontag, E., Chen, P. \& Levine, A. Transcriptional control of human p53-regulated genes. Nat. Rev. Mol. Cell Biol. 9, 402-412 (2008).

5. Ashcroft, M. \& Vousden, K. H. Regulation of p53 stability. Oncogene 18, 7637-7643 (1999).

6. Oliner, J. D., Kinzler, K. W., Meltzer, P. S., George, D. L. \& Vogelstein, B. Amplification of a gene encoding a p53-associated protein in human sarcomas. Nature 358, 80-83 (1992).

7. Wu, X., Bayle, J. H., Olson, D. \& Levine, A. J. The p53-mdm-2 autoregulatory feedback loop. Genes Dev. 7, 1126-1132 (1993).

8. el-Deiry, W. S. et al. WAF1/CIP1 is induced in p53-mediated G1 arrest and apoptosis. Cancer Res. 54, 1169-1174 (1994).

9. Miyashita, T. \& Reed, J. C. Tumor suppressor p53 is a direct transcriptional activator of the human bax gene. Cell 80, 293-299 (1995).

10. Nakano, K. \& Vousden, K. H. PUMA, a novel proapoptotic gene, is induced by p53. Mol. Cell 7, 683-694 (2001).

11. Brooks, C. L. \& Gu, W. Ubiquitination, phosphorylation and acetylation: the molecular basis for p53 regulation. Curr. Opin. Cell Biol 15, 164-171 (2003).

12. Abida, W. M., Nikolaev, A., Zhao, W., Zhang, W. \& Gu, W. FBXO11 promotes the Neddylation of $\mathrm{p} 53$ and inhibits its transcriptional activity. J. Biol. Chem. 282, 1797-1804 (2007). 
13. Xirodimas, D. P., Saville, M. K., Bourdon, J. C., Hay, R. T. \& Lane, D. P. Mdm2mediated NEDD8 conjugation of p53 inhibits its transcriptional activity. Cell 118, 83-97 (2004).

14. Gostissa, M. et al. Activation of p53 by conjugation to the ubiquitin-like protein SUMO-1. EMBO J. 18, 6462-6471 (1999).

15. Rodriguez, M. S. et al. SUMO-1 modification activates the transcriptional response of p53. EMBO J. 18, 6455-6461 (1999).

16. Huang, Y. F., Wee, S., Gunaratne, J., Lane, D. P. \& Bulavin, D. V. Isg15 controls p53 stability and functions. Cell Cycle 13, 2200-2210 (2014).

17. Haas, A. L., Ahrens, P., Bright, P. M. \& Ankel, H. Interferon induces a 15kilodalton protein exhibiting marked homology to ubiquitin. J. Biol. Chem. 262, 11315-11323 (1987).

18. Kim, M. J., Latham, A. G. \& Krug, R. M. Human influenza viruses activate an interferon-independent transcription of cellular antiviral genes: outcome with influenza A virus is unique. Proc. Natl Acad. Sci. USA 99, 10096-10101 (2002).

19. Yuan, W. \& Krug, R. M. Influenza B virus NS1 protein inhibits conjugation of the interferon (IFN)-induced ubiquitin-like ISG15 protein. EMBO J. 20, 362-371 (2001).

20. Dastur, A., Beaudenon, S., Kelley, M., Krug, R. M. \& Huibregtse, J. M. Herc5, an interferon-induced HECT E3 enzyme, is required for conjugation of ISG15 in human cells. J. Biol. Chem. 281, 4334-4338 (2006).

21. Kim, K. I., Giannakopoulos, N. V., Virgin, H. W. \& Zhang, D. E. Interferoninducible ubiquitin E2, Ubc8, is a conjugating enzyme for protein ISGylation. Mol. Cell Biol. 24, 9592-9600 (2004).

22. Zou, W. \& Zhang, D. E. The interferon-inducible ubiquitin-protein isopeptide ligase (E3) EFP also functions as an ISG15 E3 ligase. J. Biol. Chem. 281, 3989-3994 (2006).

23. Malakhov, M. P., Malakhova, O. A., Kim, K. I., Ritchie, K. J. \& Zhang, D. E. UBP43 (USP18) specifically removes ISG15 from conjugated proteins. J. Biol. Chem. 277, 9976-9981 (2002).

24. D’Cunha, J., Knight, Jr E., Haas, A. L., Truitt, R. L. \& Borden, E. C. Immunoregulatory properties of ISG15, an interferon-induced cytokine. Proc. Natl Acad. Sci. USA 93, 211-215 (1996).

25. Recht, M., Borden, E. C. \& Knight, Jr E. A human 15-kDa IFN-induced protein induces the secretion of IFN-gamma. J. Immunol. 147, 2617-2623 (1991).

26. Lai, C. et al. Mice lacking the ISG15 E1 enzyme UbE1L demonstrate increased susceptibility to both mouse-adapted and non-mouse-adapted influenza B virus infection. J. Virol. 83, 1147-1151 (2009)

27. Lenschow, D. J. et al. IFN-stimulated gene 15 functions as a critical antiviral molecule against influenza, herpes, and Sindbis viruses. Proc. Natl Acad. Sci. USA 104, 1371-1376 (2007).

28. Dauphinee, S. M. et al. Contribution of increased ISG15, ISGylation and deregulated type I IFN signaling in Usp18 mutant mice during the course of bacterial infections. Genes Immun. 15, 282-292 (2014).

29. Bogunovic, D. et al. Mycobacterial disease and impaired IFN-gamma immunity in humans with inherited ISG15 deficiency. Science 337, 1684-1688 (2012).

30. Bogunovic, D., Boisson-Dupuis, S. \& Casanova, J. L. ISG15: leading a double life as a secreted molecule. Exp. Mol. Med. 45, e18 (2013).

31. Zhang, X. et al. Human intracellular ISG15 prevents interferon-alpha/beta over-amplification and auto-inflammation. Nature 517, 89-93 (2015).

32. Jeon, Y. J. et al. Chemosensitivity is controlled by $\mathrm{p} 63$ modification with ubiquitin-like protein ISG15. J. Clin. Invest. 122, 2622-2636 (2012).

33. Park, J. M. et al. Modification of PCNA by ISG15 plays a crucial role in termination of error-prone translesion DNA synthesis. Mol. Cell 54, 626-638 (2014).

34. Sarkaria, J. N. et al. Inhibition of ATM and ATR kinase activities by the radiosensitizing agent, caffeine. Cancer Res. 59, 4375-4382 (1999).

35. el-Deiry, W. S., Kern, S. E., Pietenpol, J. A., Kinzler, K. W. \& Vogelstein, B. Definition of a consensus binding site for p53. Nat. Genet. 1, 45-49 (1992).

36. Der, S. D., Zhou, A., Williams, B. R. \& Silverman, R. H. Identification of genes differentially regulated by interferon alpha, beta, or gamma using oligonucleotide arrays. Proc. Natl Acad. Sci. USA 95, 15623-15628 (1998).

37. Ritchie, K. J. \& Zhang, D. E. ISG15: the immunological kin of ubiquitin. Semin. Cell Dev. Biol. 15, 237-246 (2004).

38. Lee, E. W. et al. Differential regulation of p53 and p21 by MKRN1 E3 ligase controls cell cycle arrest and apoptosis. EMBO J. 28, 2100-2113 (2009).

39. Gu, W. \& Roeder, R. G. Activation of p53 sequence-specific DNA binding by acetylation of the p53 C-terminal domain. Cell 90, 595-606 (1997).

40. Luo, J. et al. Acetylation of p53 augments its site-specific DNA binding both in vitro and in vivo. Proc. Natl Acad. Sci. USA 101, 2259-2264 (2004).

41. Dornan, D. \& Hupp, T. R. Inhibition of p53-dependent transcription by BOX-I phospho-peptide mimetics that bind to p300. EMBO Rep. 2, 139-144 (2001).

42. Maclaine, N. J. \& Hupp, T. R. The regulation of p53 by phosphorylation: a model for how distinct signals integrate into the p53 pathway. Aging 1, 490-502 (2009).

43. Durfee, L. A., Lyon, N., Seo, K. \& Huibregtse, J. M. The ISG15 conjugation system broadly targets newly synthesized proteins: implications for the antiviral function of ISG15. Mol. Cell 38, 722-732 (2010).
44. Leong, C. O., Vidnovic, N., DeYoung, M. P., Sgroi, D. \& Ellisen, L. W. The p63/ p73 network mediates chemosensitivity to cisplatin in a biologically defined subset of primary breast cancers. J. Clin. Invest. 117, 1370-1380 (2007).

45. Patturajan, M. et al. DeltaNp63 induces beta-catenin nuclear accumulation and signaling. Cancer Cell 1, 369-379 (2002).

46. Dohn, M., Zhang, S. \& Chen, X. p63alpha and DeltaNp63alpha can induce cell cycle arrest and apoptosis and differentially regulate p53 target genes. Oncogene 20, 3193-3205 (2001).

47. Duijf, P. H. et al. Gain-of-function mutation in ADULT syndrome reveals the presence of a second transactivation domain in p63. Hum. Mol. Genet. 11, 799-804 (2002).

48. Candi, E. et al. Differential roles of p63 isoforms in epidermal development: selective genetic complementation in p63 null mice. Cell Death Differ. 13, 1037-1047 (2006).

49. Serber, Z. et al. A C-terminal inhibitory domain controls the activity of p63 by an intramolecular mechanism. Mol. Cell. Biol. 22, 8601-8611 (2002).

50. Deutsch, G. B. et al. DNA damage in oocytes induces a switch of the quality control factor TAp63alpha from dimer to tetramer. Cell 144, 566-576 (2011)

51. Jeon, Y. J., Yoo, H. M. \& Chung, C. H. ISG15 and immune diseases. Biochim. Biophys. Acta 1802, 485-496 (2010).

52. Andersen, J. B. et al. Stage-associated overexpression of the ubiquitin-like protein, ISG15, in bladder cancer. Br. J. Cancer 94, 1465-1471 (2006).

53. Bektas, N. et al. The ubiquitin-like molecule interferon-stimulated gene 15 (ISG15) is a potential prognostic marker in human breast cancer. Breast Cancer Res. 10, R58 (2008).

54. Desai, S. D. et al. Elevated expression of ISG15 in tumor cells interferes with the ubiquitin/26S proteasome pathway. Cancer Res. 66, 921-928 (2006).

55. Kiessling, A. et al. Expression, regulation and function of the ISGylation system in prostate cancer. Oncogene 28, 2606-2620 (2009).

56. Satake, H. et al. The ubiquitin-like molecule interferon-stimulated gene 15 is overexpressed in human prostate cancer. Oncol. Rep. 23, 11-16 (2010).

57. Huang, Y. F. \& Bulavin, D. V. Oncogene-mediated regulation of p53 ISGylation and functions. Oncotarget 5, 5808-5818 (2014).

58. Chinyengetere, F. et al. Mice null for the deubiquitinase USP18 spontaneously develop leiomyosarcomas. BMC Cancer 15, 886 (2015).

59. Guo, Y. et al. Blockade of the ubiquitin protease UBP43 destabilizes transcription factor PML/RARalpha and inhibits the growth of acute promyelocytic leukemia. Cancer Res. 70, 9875-9885 (2010).

60. Guo, Y. et al. Evidence for the ubiquitin protease UBP43 as an antineoplastic target. Mol. Cancer Ther. 11, 1968-1977 (2012).

61. Shahidul Makki, M., Cristy Ruteshouser, E. \& Huff, V. Ubiquitin specific protease 18 (Usp18) is a WT1 transcriptional target. Exp. Cell Res. 319, 612-622 (2013).

62. Feng, Q. et al. UBE1L causes lung cancer growth suppression by targeting cyclin D1. Mol. Cancer Ther. 7, 3780-3788 (2008).

63. Kitareewan, S. et al. UBE1L is a retinoid target that triggers PML/RARalpha degradation and apoptosis in acute promyelocytic leukemia. Proc. Natl Acad. Sci. USA 99, 3806-3811 (2002).

64. McLaughlin, P. M. et al. The ubiquitin-activating enzyme E1-like protein in lung cancer cell lines. Int. J. Cancer 85, 871-876 (2000).

65. Pitha-Rowe, I., Hassel, B. A. \& Dmitrovsky, E. Involvement of UBE1L in ISG15 conjugation during retinoid-induced differentiation of acute promyelocytic leukemia. J. Biol. Chem. 279, 18178-18187 (2004).

66. Shah, S. J. et al. UBE1L represses PML/RAR $\{$ alpha\} by targeting the PML domain for ISG15ylation. Mol. Cancer Ther. 7, 905-914 (2008).

67. Lee, S. W. et al. SUMOylation of hnRNP-K is required for p53-mediated cell-cycle arrest in response to DNA damage. EMBO J. 31, 4441-4452 (2012).

68. Jeon, Y. J. et al. Regulation of glutamine carrier proteins by RNF5 determines breast cancer response to ER stress-inducing chemotherapies. Cancer Cell 27, 354-369 (2015).

\section{Acknowledgements}

This work was supported by the grants from the National Academy of Science of Korea, the Novel Laureate Invitation Program of Seoul National University and National Research Foundation of Korea to C.H.C. (NRF-2005-084-C00025 and M1053301000105N3301) and to J.H.S. (NRF-2014R1A2A2A01005258 and NRF-2014R1A4A1005259). J.H.P. was a recipient of BK21 fellowship.

\section{Author contributions}

J.H.P., S.W.Y., Y.J.J., J.H.S. and C.H.C. designed the research; J.H.P., S.W.Y., J.M.P., S.H.K. and J.-H.K. performed the experiment; J.H.P., S.W.Y., Y.-Y.K., Y.J.J. and J.H.S. analysed the data; J.H.P., S.W.Y., J.H.S. and C.H.C. wrote the manuscript.

\section{Additional information}

Supplementary Information accompanies this paper at http://www.nature.com/ naturecommunications 
Competing financial interests: The authors declare no competing financial interests.

Reprints and permission information is available online at http://npg.nature.com/ reprintsandpermissions/

How to cite this article: Park, J. H. et al. Positive feedback regulation of p53

transactivity by DNA damage-induced ISG15 modification. Nat. Commun. 7:12513 doi: $10.1038 /$ ncomms12513 (2016). (c) (i) This work is licensed under a Creative Commons Attribution 4.0 International License. The images or other third party material in this article are included in the article's Creative Commons license, unless indicated otherwise in the credit line; if the material is not included under the Creative Commons license, users will need to obtain permission from the license holder to reproduce the material. To view a copy of this license, visit http://creativecommons.org/licenses/by/4.0/

(C) The Author(s) 2016 